TRANSACTIONS OF THE

AMERICAN MATHEMATICAL SOCIETY

Volume 365, Number 6, June 2013, Pages 2931-2958

S 0002-9947(2012)05727-8

Article electronically published on November 30, 2012

\title{
BILINEAR DECOMPOSITIONS AND COMMUTATORS OF SINGULAR INTEGRAL OPERATORS
}

\author{
LUONG DANG KY
}

Abstract. Let $b$ be a $B M O$-function. It is well known that the linear commutator $[b, T]$ of a Calderón-Zygmund operator $T$ does not, in general, map continuously $H^{1}\left(\mathbb{R}^{n}\right)$ into $L^{1}\left(\mathbb{R}^{n}\right)$. However, Pérez showed that if $H^{1}\left(\mathbb{R}^{n}\right)$ is replaced by a suitable atomic subspace $\mathcal{H}_{b}^{1}\left(\mathbb{R}^{n}\right)$, then the commutator is continuous from $\mathcal{H}_{b}^{1}\left(\mathbb{R}^{n}\right)$ into $L^{1}\left(\mathbb{R}^{n}\right)$. In this paper, we find the largest subspace $H_{b}^{1}\left(\mathbb{R}^{n}\right)$ such that all commutators of Calderón-Zygmund operators are continuous from $H_{b}^{1}\left(\mathbb{R}^{n}\right)$ into $L^{1}\left(\mathbb{R}^{n}\right)$. Some equivalent characterizations of $H_{b}^{1}\left(\mathbb{R}^{n}\right)$ are also given. We also study the commutators $[b, T]$ for $T$ in a class $\mathcal{K}$ of sublinear operators containing almost all important operators in harmonic analysis. When $T$ is linear, we prove that there exists a bilinear operator $\mathfrak{R}=\mathfrak{R}_{T}$ mapping continuously $H^{1}\left(\mathbb{R}^{n}\right) \times B M O\left(\mathbb{R}^{n}\right)$ into $L^{1}\left(\mathbb{R}^{n}\right)$ such that for all $(f, b) \in H^{1}\left(\mathbb{R}^{n}\right) \times B M O\left(\mathbb{R}^{n}\right)$ we have

$$
[b, T](f)=\mathfrak{R}(f, b)+T(\mathfrak{S}(f, b)),
$$

where $\mathfrak{S}$ is a bounded bilinear operator from $H^{1}\left(\mathbb{R}^{n}\right) \times B M O\left(\mathbb{R}^{n}\right)$ into $L^{1}\left(\mathbb{R}^{n}\right)$ which does not depend on $T$. In the particular case of $T$ a Calderón-Zygmund operator satisfying $T 1=T^{*} 1=0$ and $b$ in $B M O^{\log }\left(\mathbb{R}^{n}\right)$, the generalized $B M O$ type space that has been introduced by Nakai and Yabuta to characterize multipliers of $B M O\left(\mathbb{R}^{n}\right)$, we prove that the commutator $[b, T]$ maps continuously $H_{b}^{1}\left(\mathbb{R}^{n}\right)$ into $h^{1}\left(\mathbb{R}^{n}\right)$. Also, if $b$ is in $B M O\left(\mathbb{R}^{n}\right)$ and $T^{*} 1=T^{*} b=0$, then the commutator $[b, T]$ maps continuously $H_{b}^{1}\left(\mathbb{R}^{n}\right)$ into $H^{1}\left(\mathbb{R}^{n}\right)$. When $T$ is sublinear, we prove that there exists a bounded subbilinear operator $\mathfrak{R}=\mathfrak{R}_{T}$ : $H^{1}\left(\mathbb{R}^{n}\right) \times B M O\left(\mathbb{R}^{n}\right) \rightarrow L^{1}\left(\mathbb{R}^{n}\right)$ such that for all $(f, b) \in H^{1}\left(\mathbb{R}^{n}\right) \times B M O\left(\mathbb{R}^{n}\right)$ we have

$(0.2) \quad|T(\mathfrak{S}(f, b))|-\mathfrak{R}(f, b) \leq|[b, T](f)| \leq \mathfrak{R}(f, b)+|T(\mathfrak{S}(f, b))|$.

The bilinear decomposition (1) and the subbilinear decomposition (2) allow us to give a general overview of all known weak and strong $L^{1}$-estimates.

\section{INTRODUCTION}

Given a function $b$ locally integrable on $\mathbb{R}^{n}$, and a Calderón-Zygmund operator $T$, we consider the linear commutator $[b, T]$ defined for smooth, compactly supported functions $f$ by

$$
[b, T](f)=b T(f)-T(b f) .
$$

A classical result of R. Coifman, R. Rochberg and G. Weiss (see [10]) states that the commutator $[b, T]$ is continuous on $L^{p}\left(\mathbb{R}^{n}\right)$ for $1<p<\infty$, when $b \in B M O\left(\mathbb{R}^{n}\right)$. Unlike the theory of Calderón-Zygmund operators, the proof of this result does not rely on a weak type $(1,1)$ estimate for $[b, T]$. In fact, it was shown in 38 that,

Received by the editors June 7, 2011.

2010 Mathematics Subject Classification. Primary 42B20; Secondary 42B30, 42B35, 42B25.

Key words and phrases. Calderón-Zygmund operators, bilinear decompositions, commutators, Hardy spaces, wavelet characterizations, BMO spaces, atoms, bilinear operators. 
in general, the linear commutator fails to be of weak type $(1,1)$, when $b$ is in $B M O\left(\mathbb{R}^{n}\right)$. Instead, an endpoint theory was provided for this operator. It is well known that any singular integral operator maps $H^{1}\left(\mathbb{R}^{n}\right)$ into $L^{1}\left(\mathbb{R}^{n}\right)$. However, it was observed in $[20$, that the commutator $[b, H]$ with $b$ in $B M O(\mathbb{R})$, where $H$ is a Hilbert transform on $\mathbb{R}$, does not map, in general, $H^{1}(\mathbb{R})$ into $L^{1}(\mathbb{R})$. Instead of this, the weak type estimate $\left(H^{1}, L^{1}\right)$ for $[b, T]$ is well known; see for example [27, 32, 43]. Observe that intuitively one would like to write

$$
[b, T](f)=\sum_{j=1}^{\infty} \lambda_{j}\left(b-b_{B_{j}}\right) T\left(a_{j}\right)-T\left(\sum_{j=1}^{\infty} \lambda_{j}\left(b-b_{B_{j}}\right) a_{j}\right),
$$

where $f=\sum_{j=1}^{\infty} \lambda_{j} a_{j}$ is an atomic decomposition of $f$ and $b_{B_{j}}$ is the average of $b$ on $B_{j}$. This is equivalent to asking for a commutation property

$$
\sum_{j=1}^{\infty} \lambda_{j} b_{B_{j}} T\left(a_{j}\right)=T\left(\sum_{j=1}^{\infty} \lambda_{j} b_{B_{j}} a_{j}\right) .
$$

Even if most authors, for instance in [27, 32, 43, 45, 25, 42, 26, implicitly use (1.1), one must be careful at this point. Indeed, equality (1.1) is not clear since the two series $\sum_{j=1}^{\infty} \lambda_{j} b_{B_{j}} T\left(a_{j}\right)$ and $\sum_{j=1}^{\infty} \lambda_{j} b_{B_{j}} a_{j}$ are not yet well defined, in general. We refer the reader to [6], Section 3 , to be convinced that one must be careful with equality (1.1).

Although the commutator $[b, T]$ does not map continuously, in general, $H^{1}\left(\mathbb{R}^{n}\right)$ into $L^{1}\left(\mathbb{R}^{n}\right)$, following Pérez 38 , one can find a subspace $\mathcal{H}_{b}^{1}\left(\mathbb{R}^{n}\right)$ of $H^{1}\left(\mathbb{R}^{n}\right)$ such that $[b, T]$ maps continuously $\mathcal{H}_{b}^{1}\left(\mathbb{R}^{n}\right)$ into $L^{1}\left(\mathbb{R}^{n}\right)$. Recall that (see [38]) a function $a$ is a $b$-atom if

i) supp $a \subset Q$ for some cube $Q$,

ii) $\|a\|_{L^{\infty}} \leq|Q|^{-1}$

iii) $\int_{\mathbb{R}^{n}} a(x) d x=\int_{\mathbb{R}^{n}} a(x) b(x) d x=0$.

The space $\mathcal{H}_{b}^{1}\left(\mathbb{R}^{n}\right)$ consists of the subspace of $L^{1}\left(\mathbb{R}^{n}\right)$ of functions $f$ which can be written as $f=\sum_{j=1}^{\infty} \lambda_{j} a_{j}$, where the $a_{j}$ 's are $b$-atoms, and the $\lambda_{j}$ 's are complex numbers with $\sum_{j=1}^{\infty}\left|\lambda_{j}\right|<\infty$.

In [38] the author showed that the commutator $[b, T]$ is bounded from $\mathcal{H}_{b}^{1}\left(\mathbb{R}^{n}\right)$ into $L^{1}\left(\mathbb{R}^{n}\right)$ by establishing that

$$
\sup \left\{\|[b, T](a)\|_{L^{1}}: a \text { is a } b \text {-atom }\right\}<\infty .
$$

This leaves a gap in the proof which we fill here (see below). Indeed, as is pointed out in [6], there exists a linear operator $U$ defined on the space of all finite linear combinations of $(1, \infty)$-atoms satisfying

$$
\sup \left\{\|U(a)\|_{L^{1}}: a \text { is a }(1, \infty)-\text { atom }\right\}<\infty,
$$

but which does not admit an extension to a bounded operator from $H^{1}\left(\mathbb{R}^{n}\right)$ into $L^{1}\left(\mathbb{R}^{n}\right)$. From this result, we see that inequality (1.2) does not suffice to conclude that $[b, T]$ is bounded from $\mathcal{H}_{b}^{1}\left(\mathbb{R}^{n}\right)$ into $L^{1}\left(\mathbb{R}^{n}\right)$. In the setting of $H^{1}\left(\mathbb{R}^{n}\right)$, it is well known (see [34 or 44] for details) that a linear operator $U$ can be extended to a bounded operator from $H^{1}\left(\mathbb{R}^{n}\right)$ into $L^{1}\left(\mathbb{R}^{n}\right)$ if for some $1<q<\infty$, we have

$$
\sup \left\{\|U(a)\|_{L^{1}}: a \text { is a }(1, q)-\text { atom }\right\}<\infty .
$$

It follows from the fact that the finite atomic norm on $H_{\text {fin }}^{1, q}\left(\mathbb{R}^{n}\right)$ is equivalent to the standard infinite atomic decomposition norm on $H_{\text {ato }}^{1, q}\left(\mathbb{R}^{n}\right)$ through the grand 
maximal function characterization of $H^{1}\left(\mathbb{R}^{n}\right)$. However, one cannot use this method in the context of $\mathcal{H}_{b}^{1}\left(\mathbb{R}^{n}\right)$.

Also, a natural question arises: can one find the largest subspace of $H^{1}\left(\mathbb{R}^{n}\right.$ ) (of course, this space contains $\mathcal{H}_{b}^{1}\left(\mathbb{R}^{n}\right)$; see also Theorem [5.2) such that all commutators $[b, T]$ of Calderón-Zygmund operators are bounded from this space into $L^{1}\left(\mathbb{R}^{n}\right)$ ? For $b \in B M O\left(\mathbb{R}^{n}\right)$, a non-constant function, we consider the space $H_{b}^{1}\left(\mathbb{R}^{n}\right)$ consisting of all $f \in H^{1}\left(\mathbb{R}^{n}\right)$ such that the (sublinear) commutator $[b, \mathfrak{M}]$ of $f$ belongs to $L^{1}\left(\mathbb{R}^{n}\right)$, where $\mathfrak{M}$ is the non-tangential grand maximal operator (see Section 2). The norm on $H_{b}^{1}\left(\mathbb{R}^{n}\right)$ is defined by $\|f\|_{H_{b}^{1}}:=\|f\|_{H^{1}}\|b\|_{B M O}+\|[b, \mathfrak{M}](f)\|_{L^{1}}$. Here we just consider non-constant $B M O$-functions $b$ since the commutator $[b, T]=0$ if $b$ is a constant function. Then, we prove that $[b, T]$ is bounded from $H_{b}^{1}\left(\mathbb{R}^{n}\right)$ into $L^{1}\left(\mathbb{R}^{n}\right)$ for every Calderón-Zygmund singular integral operator $T$ (in fact it holds for all $T \in \mathcal{K}$; see below). Furthermore, $H_{b}^{1}\left(\mathbb{R}^{n}\right)$ is the largest space having this property (see Remark 5.1). This answers the question above. Besides, we also consider the class $\mathcal{K}$ of all sublinear operators $T$, bounded from $H^{1}\left(\mathbb{R}^{n}\right)$ into $L^{1}\left(\mathbb{R}^{n}\right)$, satisfying the condition

$$
\left\|\left(b-b_{Q}\right) T a\right\|_{L^{1}} \leq C\|b\|_{B M O}
$$

for all $B M O$-functions $b$, where the $H^{1}$-atom $a$ is related to the cube $Q$. Here $b_{Q}$ denotes the average of $b$ on $Q$, and $C>0$ is a constant independent of $b, a$. This class $\mathcal{K}$ contains almost all important operators in harmonic analysis: Calderón-Zygmund type operators, strongly singular integral operators, multiplier operators, pseudodifferential operators, maximal type operators, the area integral operator of Lusin, Littlewood-Paley type operators, Marcinkiewicz operators, maximal Bochner-Riesz operators, etc. (see Section 4). When $T$ is linear and belongs to $\mathcal{K}$, we prove that there exists a bounded bilinear operator $\mathfrak{R}=\mathfrak{R}_{T}: H^{1}\left(\mathbb{R}^{n}\right) \times B M O\left(\mathbb{R}^{n}\right) \rightarrow$ $L^{1}\left(\mathbb{R}^{n}\right)$ such that for all $(f, b) \in H^{1}\left(\mathbb{R}^{n}\right) \times B M O\left(\mathbb{R}^{n}\right)$ we have the following bilinear decomposition:

$$
[b, T](f)=\mathfrak{R}(f, b)+T(\mathfrak{S}(f, b)),
$$

where $\mathfrak{S}$ is a bounded bilinear operator from $H^{1}\left(\mathbb{R}^{n}\right) \times B M O\left(\mathbb{R}^{n}\right)$ into $L^{1}\left(\mathbb{R}^{n}\right)$ which does not depend on $T$ (see Section 3 ). This bilinear decomposition is strongly related to our previous result in [4] on the paraproduct and product on $H^{1}\left(\mathbb{R}^{n}\right) \times$ $B M O\left(\mathbb{R}^{n}\right)$.

We then prove that $[b, T]$ is bounded from $H_{b}^{1}\left(\mathbb{R}^{n}\right)$ into $L^{1}\left(\mathbb{R}^{n}\right)$ (see Theorem 3.3) via the bilinear decomposition (1.3) (see Theorem 3.2) and some characterizations of $H_{b}^{1}\left(\mathbb{R}^{n}\right.$ ) (see Theorem 5.1). Furthermore, by using the weak convergence theorem in $H^{1}\left(\mathbb{R}^{n}\right)$ of Jones and Journé, we prove that $\mathcal{H}_{b}^{1}\left(\mathbb{R}^{n}\right) \subset H_{b}^{1}\left(\mathbb{R}^{n}\right)$ (see Theorem 5.2). These allow us to fill the gap mentioned above in [38.

On the other hand, as an immediate corollary of the bilinear decomposition (1.3), we also obtain the weak type estimate $\left(H^{1}, L^{1}\right)$ for the commutator $[b, T]$, where $T$ is a Calderón-Zygmund type operator, a strongly singular integral operator, a multiplier operator or a pseudo-differential operator. We also point out that weak type estimates and Hardy type estimates for the (linear) commutators of multiplier operators and of strongly singular Calderón-Zygmund operators have been studied recently (see [45, 25, 42] for the multiplier operators and [26] for strongly singular Calderón-Zygmund operators). 
Next, two natural questions for Hardy-type estimates of the commutator $[b, T]$ arose: when does $[b, T]$ map $H_{b}^{1}\left(\mathbb{R}^{n}\right)$ into $h^{1}\left(\mathbb{R}^{n}\right)$ and when does $[b, T]$ map $H_{b}^{1}\left(\mathbb{R}^{n}\right)$ into $H^{1}\left(\mathbb{R}^{n}\right)$ ?

This paper gives two sufficient conditions for the above two questions. Recall that $B M O^{\log }\left(\mathbb{R}^{n}\right)$, the generalized $B M O$ type space that has been introduced by Nakai and Yabuta 37 to characterize multipliers of $B M O\left(\mathbb{R}^{n}\right)$, is the space of all locally integrable functions $f$ such that

$$
\|f\|_{B M O^{\log }}:=\sup _{B(a, r)} \frac{|\log r|+\log (e+|a|)}{|B(a, r)|} \int_{B(a, r)}\left|f(x)-f_{B(a, r)}\right| d x<\infty .
$$

We obtain that if $T$ is a Calderón-Zygmund operator satisfying $T 1=T^{*} 1=0$ and $b$ is in $B M O^{\log }\left(\mathbb{R}^{n}\right)$, then the linear commutator $[b, T]$ maps continuously $H_{b}^{1}\left(\mathbb{R}^{n}\right)$ into $h^{1}\left(\mathbb{R}^{n}\right)$. This gives a sufficient condition to the first problem. For the second one, we prove that if $T$ is a Calderón-Zygmund operator satisfying $T^{*} 1=T^{*} b=0$ and $b$ is in $B M O\left(\mathbb{R}^{n}\right)$, then the linear commutator $[b, T]$ maps continuously $H_{b}^{1}\left(\mathbb{R}^{n}\right)$ into $H^{1}\left(\mathbb{R}^{n}\right)$.

A difficult point to prove the first result is that we have to deal directly with $f \in H_{b}^{1}\left(\mathbb{R}^{n}\right)$. It would be easier to do it for atomic type Hardy spaces as in the case of $\mathcal{H}_{b}^{1}\left(\mathbb{R}^{n}\right)$. However, we do not know whether there exists an atomic characterization for the space $H_{b}^{1}\left(\mathbb{R}^{n}\right)$. This is still an open question.

Let $X$ be a Banach space. We say that an operator $T: X \rightarrow L^{1}\left(\mathbb{R}^{n}\right)$ is a sublinear operator if for all $f, g \in X$ and $\alpha, \beta \in \mathbb{C}$, we have

$$
|T(\alpha f+\beta g)(x)| \leq|\alpha||T f(x)|+|\beta||T g(x)| .
$$

Obviously, a linear operator $T: X \rightarrow L^{1}\left(\mathbb{R}^{n}\right)$ is a sublinear operator. We also say that an operator $\mathfrak{T}: H^{1}\left(\mathbb{R}^{n}\right) \times B M O\left(\mathbb{R}^{n}\right) \rightarrow L^{1}\left(\mathbb{R}^{n}\right)$ is a subbilinear operator if for all $(f, g) \in H^{1}\left(\mathbb{R}^{n}\right) \times B M O\left(\mathbb{R}^{n}\right)$ the operators $\mathfrak{T}(f, \cdot): B M O\left(\mathbb{R}^{n}\right) \rightarrow L^{1}\left(\mathbb{R}^{n}\right)$ and $\mathfrak{T}(\cdot, g): H^{1}\left(\mathbb{R}^{n}\right) \rightarrow L^{1}\left(\mathbb{R}^{n}\right)$ are sublinear operators.

In this paper, we also obtain the subbilinear decomposition for sublinear commutators. More precisely, when $T \in \mathcal{K}$ is a sublinear operator, we prove that there exists a bounded subbilinear operator $\mathfrak{R}=\mathfrak{R}_{T}: H^{1}\left(\mathbb{R}^{n}\right) \times B M O\left(\mathbb{R}^{n}\right) \rightarrow L^{1}\left(\mathbb{R}^{n}\right)$ so that for all $(f, b) \in H^{1}\left(\mathbb{R}^{n}\right) \times B M O\left(\mathbb{R}^{n}\right)$, we have

$$
|T(\mathfrak{S}(f, b))|-\mathfrak{R}(f, b) \leq|[b, T](f)| \leq \mathfrak{R}(f, b)+|T(\mathfrak{S}(f, b))| .
$$

Then, the strong type estimate $\left(H_{b}^{1}, L^{1}\right)$ and the weak type estimate $\left(H^{1}, L^{1}\right)$ of the commutators of Littlewood-Paley type operators, of Marcinkiewicz operators, and of maximal Bochner-Riesz operators, can be viewed as an immediate corollary of (1.4). When $H_{b}^{1}\left(\mathbb{R}^{n}\right)$ is replaced by $\mathcal{H}_{b}^{1}\left(\mathbb{R}^{n}\right)$, these types of estimates have also been considered recently (see for example [28, 7, 33, 30, 31, 29]).

Let us emphasize the three main purposes of this paper. First, we want to give the bilinear (resp., subbilinear) decompositions for the linear (resp., sublinear) commutators. Second, we find the largest subspace of $H^{1}\left(\mathbb{R}^{n}\right)$ such that all commutators of Calderón-Zygmund operators map continuously this space into $L^{1}\left(\mathbb{R}^{n}\right)$. Finally, we obtain the $\left(H_{b}^{1}, h^{1}\right)$ and $\left(H_{b}^{1}, H^{1}\right)$ type estimates for commutators of Calderón-Zygmund operators.

Our paper is organized as follows. In Section 2 we present some notation and preliminaries about the Calderón-Zygmund operators, the function spaces we use, and a short introduction to wavelets, a useful tool in our work. In Section 3 we state our two decomposition theorems (Theorem 3.1 and Theorem 3.2 ), the $\left(H_{b}^{1}, L^{1}\right)$ 
type estimates for commutators (Theorem 3.3), and some remarks. The bilinear type estimates for commutators of Calderón-Zygmund operators (Theorem 3.4) and the boundedness of commutators of Calderón-Zygmund operators on Hardy spaces are also given in this section. In Section 4 we give some examples of operators in the class $\mathcal{K}$ and recall our result from [4] which decomposes a product of $f$ in $H^{1}\left(\mathbb{R}^{n}\right)$ and $g$ in $B M O\left(\mathbb{R}^{n}\right)$ as a sum of images by four bilinear operators defined through wavelets. These operators are fundamental for the two decomposition theorems. In Section 5 we study the space $H_{b}^{1}\left(\mathbb{R}^{n}\right)$. Sections 6 and 7 are devoted to the proofs of the two decomposition theorems, the $\left(H_{b}^{1}, L^{1}\right)$ type estimates of commutators $[b, T]$ with $T \in \mathcal{K}$, and the boundedness results of commutators of Calderón-Zygmund operators. Finally, in Section 8 we present without proofs some results for commutators of fractional integrals.

Throughout the whole paper, $C$ denotes a positive geometric constant which is independent of the main parameters, but may change from line to line. In $\mathbb{R}^{n}$, we denote by $Q=Q[x, r]:=\left\{y=\left(y_{1}, \ldots, y_{n}\right) \in \mathbb{R}^{n}: \sup _{1 \leq i \leq n}\left|y_{i}-x_{i}\right| \leq r\right\}$ a cube with center $x=\left(x_{1}, \ldots, x_{n}\right)$ and radius $r>0$. For any measurable set $E$, we denote by $\chi_{E}$ its characteristic function, by $|E|$ its Lebesgue measure, and by $E^{c}$ the set $\mathbb{R}^{n} \backslash E$. For a cube $Q$ and $f$ a locally integrable function, we denote by $f_{Q}$ the average of $f$ on $Q$.

\section{Some PRELIMINARIES AND NOTATION}

As usual, $\mathcal{S}\left(\mathbb{R}^{n}\right)$ denotes the Schwartz class of test functions on $\mathbb{R}^{n}, \mathcal{S}^{\prime}\left(\mathbb{R}^{n}\right)$ the space of tempered distributions, and $C_{0}^{\infty}\left(\mathbb{R}^{n}\right)$ the space of $C^{\infty}$-functions with compact support.

2.1. Calderón-Zygmund operators. Let $\delta \in(0,1]$. A continuous function $K$ : $\mathbb{R}^{n} \times \mathbb{R}^{n} \backslash\left\{(x, x): x \in \mathbb{R}^{n}\right\} \rightarrow \mathbb{C}$ is said to be a $\delta$-Calderón-Zygmund singular integral kernel if there exists a constant $C>0$ such that

for all $x \neq y$, and

$$
|K(x, y)| \leq \frac{C}{|x-y|^{n}}
$$

$$
\left|K(x, y)-K\left(x^{\prime}, y\right)\right|+\left|K(y, x)-K\left(y, x^{\prime}\right)\right| \leq C \frac{\left|x-x^{\prime}\right|^{\delta}}{|x-y|^{n+\delta}}
$$

for all $2\left|x-x^{\prime}\right| \leq|x-y|$.

A linear operator $T: \mathcal{S}\left(\mathbb{R}^{n}\right) \rightarrow \mathcal{S}^{\prime}\left(\mathbb{R}^{n}\right)$ is said to be a $\delta$-Calderón-Zygmund operator if $T$ can be extended to a bounded operator on $L^{2}\left(\mathbb{R}^{n}\right)$ and if there exists a $\delta$-Calderón-Zygmund singular integral kernel $K$ such that for all $f \in C_{0}^{\infty}\left(\mathbb{R}^{n}\right)$ and all $x \notin \operatorname{supp} f$, we have

$$
T f(x)=\int_{\mathbb{R}^{n}} K(x, y) f(y) d y .
$$

We say that $T$ is a Calderón-Zygmund operator if it is a $\delta$-Calderón-Zygmund operator for some $\delta \in(0,1]$.

We say that the Calderón-Zygmund operator $T$ satisfies the condition $T^{*} 1=0$ (resp., $T 1=0$ ) if $\int_{\mathbb{R}^{n}} T a(x) d x=0$ (resp., $\int_{\mathbb{R}^{n}} T^{*} a(x) d x=0$ ) holds for all classical $H^{1}$-atoms $a$. Let $b$ be a locally integrable function on $\mathbb{R}^{n}$. We say that the CalderónZygmund operator $T$ satisfies the condition $T^{*} b=0$ if $\int_{\mathbb{R}^{n}} b(x) T a(x) d x=0$ holds for all classical $H^{1}$-atoms $a$. 
2.2. Function spaces. We first consider the subspace $\mathcal{A}$ of $\mathcal{S}\left(\mathbb{R}^{n}\right)$ defined by

$$
\mathcal{A}=\left\{\phi \in \mathcal{S}\left(\mathbb{R}^{n}\right):|\phi(x)|+|\nabla \phi(x)| \leq\left(1+|x|^{2}\right)^{-(n+1)}\right\},
$$

where $\nabla=\left(\partial / \partial x_{1}, \ldots, \partial / \partial x_{n}\right)$ denotes the gradient. We then define

$$
\mathfrak{M} f(x):=\sup _{\phi \in \mathcal{A}} \sup _{|y-x|<t}\left|f * \phi_{t}(y)\right| \quad \text { and } \mathfrak{m} f(x):=\sup _{\phi \in \mathcal{A}} \sup _{|y-x|<t<1}\left|f * \phi_{t}(y)\right|,
$$

where $\phi_{t}(\cdot)=t^{-n} \phi\left(t^{-1} \cdot\right)$. The space $H^{1}\left(\mathbb{R}^{n}\right)$ is the space of all tempered distributions $f$ such that $\mathfrak{M} f \in L^{1}\left(\mathbb{R}^{n}\right)$ equipped with the norm $\|f\|_{H^{1}}=\|\mathfrak{M} f\|_{L^{1}}$. The space $h^{1}\left(\mathbb{R}^{n}\right)$ denotes the space of all tempered distributions $f$ such that $\mathfrak{m} f \in L^{1}\left(\mathbb{R}^{n}\right)$ equipped with the norm $\|f\|_{h^{1}}=\|\mathfrak{m} f\|_{L^{1}}$. The space $H^{\log }\left(\mathbb{R}^{n}\right)$ (see $[23,4])$ denotes the space of all tempered distributions $f$ such that $\mathfrak{M} f \in L^{\log }\left(\mathbb{R}^{n}\right)$

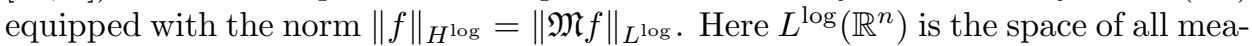
surable functions $f$ such that $\int_{\mathbb{R}^{n}} \frac{|f(x)|}{\log (e+|x|)+\log (e+|f(x)|)} d x<\infty$ with the (quasi-) norm

$$
\|f\|_{L^{\log }}:=\inf \left\{\lambda>0: \int_{\mathbb{R}^{n}} \frac{\frac{|f(x)|}{\lambda}}{\log (e+|x|)+\log \left(e+\frac{|f(x)|}{\lambda}\right)} d x \leq 1\right\} .
$$

Clearly, for any $f \in H^{1}\left(\mathbb{R}^{n}\right)$, we have

$$
\|f\|_{h^{1}} \leq\|f\|_{H^{1}} \text { and } \quad\|f\|_{H^{\log }} \leq\|f\|_{H^{1}} .
$$

We remark that the local real Hardy space $h^{1}\left(\mathbb{R}^{n}\right)$, first introduced by Goldberg [18], is larger than $H^{1}\left(\mathbb{R}^{n}\right)$ and allows more flexibility, since global cancellation conditions are not necessary. For example, the Schwartz space is contained in $h^{1}\left(\mathbb{R}^{n}\right)$ but not in $H^{1}\left(\mathbb{R}^{n}\right)$, and multiplication by cutoff functions preserves $h^{1}\left(\mathbb{R}^{n}\right)$ but not $H^{1}\left(\mathbb{R}^{n}\right)$. Thus it makes $h^{1}\left(\mathbb{R}^{n}\right)$ more suitable for working in domains and on manifolds.

It is well known (see 15 or 40 ) that the dual of $H^{1}\left(\mathbb{R}^{n}\right)$ is $B M O\left(\mathbb{R}^{n}\right)$, the space of all locally integrable functions $f$ with

$$
\|f\|_{B M O}:=\sup _{B} \frac{1}{|B|} \int_{B}\left|f(x)-f_{B}\right| d x<\infty
$$

where the supremum is taken over all balls $B$. We note $\mathbb{Q}:=[0,1)^{n}$ and, for $f$ a function in $B M O\left(\mathbb{R}^{n}\right)$,

$$
\|f\|_{B M O^{+}}:=\|f\|_{B M O}+\left|f_{\mathbb{Q}}\right| .
$$

We should also point out that the space $H^{\log }\left(\mathbb{R}^{n}\right)$ arises naturally in the study of pointwise products of functions in $H^{1}\left(\mathbb{R}^{n}\right)$ with functions in $B M O\left(\mathbb{R}^{n}\right)$, and in the endpoint estimates for the div-curl lemma (see for example [3, 4, 23]).

In [18] it was shown that the dual of $h^{1}\left(\mathbb{R}^{n}\right)$ can be identified with the space $\operatorname{bmo}\left(\mathbb{R}^{n}\right)$, consisting of locally integrable functions $f$ with

$$
\|f\|_{b m o}:=\sup _{|B| \leq 1} \frac{1}{|B|} \int_{B}\left|f(x)-f_{B}\right| d x+\sup _{|B| \geq 1} \frac{1}{|B|} \int_{B}|f(x)| d x<\infty,
$$

where the supremums are taken over all balls $B$.

Clearly, for any $f \in b m o\left(\mathbb{R}^{n}\right)$, we have

$$
\|f\|_{B M O} \leq\|f\|_{B M O^{+}} \leq C\|f\|_{b m o} .
$$

We next recall that the space $V M O\left(\mathbb{R}^{n}\right)$ (resp., vmo $\left(\mathbb{R}^{n}\right)$ ) is the closure of $C_{0}^{\infty}\left(\mathbb{R}^{n}\right)$ in $\left(B M O\left(\mathbb{R}^{n}\right),\|\cdot\|_{B M O}\right)$ (resp., $\left.\left(b m o\left(\mathbb{R}^{n}\right),\|\cdot\|_{b m o}\right)\right)$. It is well known 
that (see [9] and 11]) the dual of $V M O\left(\mathbb{R}^{n}\right)$ (resp., vmo $\left.\left(\mathbb{R}^{n}\right)\right)$ is the Hardy space $H^{1}\left(\mathbb{R}^{n}\right)$ (resp., $\left.h^{1}\left(\mathbb{R}^{n}\right)\right)$. We point out that the space $V M O\left(\mathbb{R}^{n}\right)\left(\right.$ resp., vmo $\left(\mathbb{R}^{n}\right)$ ) considered by Coifman and Weiss (resp., Dafni [11]) is different from the one considered by Sarason. Thanks to Bourdaud [5], it coincides with the space $\operatorname{VMO}\left(\mathbb{R}^{n}\right)$ (resp., vmo $\left(\mathbb{R}^{n}\right)$ ) considered above.

In the study of the pointwise multipliers for $B M O\left(\mathbb{R}^{n}\right)$, Nakai and Yabuta 37 introduced the space $B M O^{\log }\left(\mathbb{R}^{n}\right)$, consisting of locally integrable functions $f$ with

$$
\|f\|_{B M O^{\log }}:=\sup _{B(a, r)} \frac{|\log r|+\log (e+|a|)}{|B(a, r)|} \int_{B(a, r)}\left|f(x)-f_{B(a, r)}\right| d x<\infty .
$$

There, the authors proved that a function $g$ is a pointwise multiplier for $B M O\left(\mathbb{R}^{n}\right)$ if and only if $g$ belongs to $L^{\infty}\left(\mathbb{R}^{n}\right) \cap B M O^{\log }\left(\mathbb{R}^{n}\right)$. Furthermore, it is also shown in 23. that the space $B M O^{\log }\left(\mathbb{R}^{n}\right)$ is the dual of the space $H^{\log }\left(\mathbb{R}^{n}\right)$.

Definition 2.1. Let $b$ be a locally integrable function and $1<q \leq \infty$. A function $a$ is called a $(q, b)$-atom related to the cube $Q$ if

i) supp $a \subset Q$,

ii) $\|a\|_{L^{q}} \leq|Q|^{1 / q-1}$,

iii) $\int_{\mathbb{R}^{n}} a(x) d x=\int_{\mathbb{R}^{n}} a(x) b(x) d x=0$.

The space $\mathcal{H}_{b}^{1, q}\left(\mathbb{R}^{n}\right)$ consists of the subspace of $L^{1}\left(\mathbb{R}^{n}\right)$ of functions $f$ which can be written as $f=\sum_{j=1}^{\infty} \lambda_{j} a_{j}$, where the $a_{j}$ 's are $(q, b)$-atoms, $\lambda_{j} \in \mathbb{C}$, and $\sum_{j=1}^{\infty}\left|\lambda_{j}\right|<\infty$. As usual, we define on $\mathcal{H}_{b}^{1, q}\left(\mathbb{R}^{n}\right)$ the norm

$$
\|f\|_{\mathcal{H}_{b}^{1, q}}:=\inf \left\{\sum_{j=1}^{\infty}\left|\lambda_{j}\right|: f=\sum_{j=1}^{\infty} \lambda_{j} a_{j}\right\} .
$$

Observe that when $q=\infty$, then the space $\mathcal{H}_{b}^{1, \infty}\left(\mathbb{R}^{n}\right)$ is just the space $\mathcal{H}_{b}^{1}\left(\mathbb{R}^{n}\right)$ considered in 38. Furthermore, $\mathcal{H}_{b}^{1, \infty}\left(\mathbb{R}^{n}\right) \subset \mathcal{H}_{b}^{1, q}\left(\mathbb{R}^{n}\right) \subset H^{1}\left(\mathbb{R}^{n}\right)$ and the inclusions are continuous.

We next introduce the space $H_{b}^{1}\left(\mathbb{R}^{n}\right)$ as follows.

Definition 2.2. Let $b$ be a non-constant $B M O$-function. The space $H_{b}^{1}\left(\mathbb{R}^{n}\right)$ consists of all $f$ in $H^{1}\left(\mathbb{R}^{n}\right)$ such that $[b, \mathfrak{M}](f)(x)=\mathfrak{M}(b(x) f(\cdot)-b(\cdot) f(\cdot))(x)$ belongs to $L^{1}\left(\mathbb{R}^{n}\right)$. We equipped $H_{b}^{1}\left(\mathbb{R}^{n}\right)$ with the norm $\|f\|_{H_{b}^{1}}:=\|f\|_{H^{1}}\|b\|_{B M O}+$ $\|[b, \mathfrak{M}](f)\|_{L^{1}}$.

2.3. Prerequisites on wavelets. Let us consider a wavelet basis of $\mathbb{R}$ with compact support. More explicitly, we are first given a $\mathcal{C}^{1}(\mathbb{R})$-wavelet in dimension one, called $\psi$, such that $\left\{2^{j / 2} \psi\left(2^{j} x-k\right)\right\}_{j, k \in \mathbb{Z}}$ form an $L^{2}(\mathbb{R})$ basis. We assume that this wavelet basis comes for a multiresolution analysis (MRA) on $\mathbb{R}$, as defined below (see [35]).

Definition 2.3. A multiresolution analysis (MRA) on $\mathbb{R}$ is defined as an increasing sequence $\left\{V_{j}\right\}_{j \in \mathbb{Z}}$ of closed subspaces of $L^{2}(\mathbb{R})$ with the following four properties:

i) $\bigcap_{j \in \mathbb{Z}} V_{j}=\{0\}$ and $\overline{\bigcup_{j \in \mathbb{Z}} V_{j}}=L^{2}(\mathbb{R})$,

ii) for every $f \in L^{2}(\mathbb{R})$ and every $j \in \mathbb{Z}, f(x) \in V_{j}$ if and only if $f(2 x) \in V_{j+1}$,

iii) for every $f \in L^{2}(\mathbb{R})$ and every $k \in \mathbb{Z}, f(x) \in V_{0}$ if and only if $f(x-k) \in V_{0}$,

iv) there exists a function $\phi \in L^{2}(\mathbb{R})$, called the scaling function, such that the family $\left\{\phi_{k}(x)=\phi(x-k): k \in \mathbb{Z}\right\}$ is an orthonormal basis for $V_{0}$. 
It is classical that, given an (MRA) on $\mathbb{R}$, one can find a wavelet $\psi$ such that $\left\{2^{j / 2} \psi\left(2^{j} x-k\right)\right\}_{k \in \mathbb{Z}}$ is an orthonormal basis of $W_{j}$, the orthogonal complement of $V_{j}$ in $V_{j+1}$. Moreover, by Daubechies Theorem (see [12]), it is possible to find a suitable (MRA) so that $\phi$ and $\psi$ are $\mathcal{C}^{1}(\mathbb{R})$ and compactly supported, $\psi$ has mean 0 and $\int x \psi(x) d x=0$, which is known as the moment condition. We could content ourselves, in the following theorems, to have $\phi$ and $\psi$ decreasing sufficiently rapidly at $\infty$, but proofs are simpler with compactly supported wavelets. More precisely we can choose $m>1$ such that $\phi$ and $\psi$ are supported in the interval $1 / 2+m(-1 / 2,+1 / 2)$, which is obtained from $(0,1)$ by a dilation by $m$ centered at $1 / 2$.

Going back to $\mathbb{R}^{n}$, we recall that a wavelet basis of $\mathbb{R}^{n}$ is constructed as follows. We call $E$ the set $E=\{0,1\}^{n} \backslash\{(0, \ldots, 0)\}$ and, for $\sigma \in E$, put $\psi^{\sigma}(x)=$ $\phi^{\sigma_{1}}\left(x_{1}\right) \cdots \phi^{\sigma_{n}}\left(x_{n}\right)$, with $\phi^{\sigma_{j}}\left(x_{j}\right)=\phi\left(x_{j}\right)$ for $\sigma_{j}=0$ while $\phi^{\sigma_{j}}\left(x_{j}\right)=\psi\left(x_{j}\right)$ for $\sigma_{j}=1$. Then the set $\left\{2^{n j / 2} \psi^{\sigma}\left(2^{j} x-k\right)\right\}_{j \in \mathbb{Z}, k \in \mathbb{Z}^{n}, \sigma \in E}$ is an orthonormal basis of $L^{2}\left(\mathbb{R}^{n}\right)$. As is classical, for $I$ a dyadic cube of $\mathbb{R}^{n}$, which may be written as the set of $x$ such that $2^{j} x-k \in(0,1)^{n}$, we note

$$
\psi_{I}^{\sigma}(x)=2^{n j / 2} \psi^{\sigma}\left(2^{j} x-k\right) .
$$

We also note $\phi_{I}=2^{n j / 2} \phi_{(0,1)^{n}}\left(2^{j} x-k\right)$, with $\phi_{(0,1)^{n}}$ the scaling function in $n$ variables, given by $\phi_{(0,1)^{n}}(x)=\phi\left(x_{1}\right) \cdots \phi\left(x_{n}\right)$. In the sequel, the letter $I$ always refers to dyadic cubes. Moreover, we denote by $k I$ the cube of the same center dilated by the coefficient $k$. Because of the assumption on the supports of $\phi$ and $\psi$, the functions $\psi_{I}^{\sigma}$ and $\phi_{I}$ are supported in the cube $m I$.

The wavelet basis $\left\{\psi_{I}^{\sigma}\right\}$, obtained by letting $I$ vary among dyadic cubes and $\sigma$ in $E$, comes from an (MRA) in $\mathbb{R}^{n}$, which we still denote by $\left\{V_{j}\right\}_{j \in \mathbb{Z}}$, obtained by taking tensor products of the one-dimensional ones.

The following theorem gives the wavelet characterization of $H^{1}\left(\mathbb{R}^{n}\right)$ (cf. [35, 21] ).

Theorem 2.1. There exists a constant $C>0$ such that $f \in H^{1}\left(\mathbb{R}^{n}\right)$ if and only if

$$
\begin{gathered}
\mathcal{W}_{\psi} f:=\left(\sum_{I} \sum_{\sigma \in E}\left|\left\langle f, \psi_{I}^{\sigma}\right\rangle\right|^{2}|I|^{-1} \chi_{I}\right)^{1 / 2} \in L^{1}\left(\mathbb{R}^{n}\right) ; \text { moreover }, \\
C^{-1}\|f\|_{H^{1}} \leq\left\|\mathcal{W}_{\psi} f\right\|_{L^{1}} \leq C\|f\|_{H^{1}} .
\end{gathered}
$$

A function $a \in L^{2}\left(\mathbb{R}^{n}\right)$ is called a $\psi$-atom related to the (not necessarily dyadic) cube $R$ if it may be written as

$$
a=\sum_{I \subset R} \sum_{\sigma \in E} a_{I, \sigma} \psi_{I}^{\sigma}
$$

with $\|a\|_{L^{2}} \leq|R|^{-1 / 2}$. Observe that $a$ is compactly supported in $m R$ and has mean 0 , so that it is a classical atom related to $m R$, up to the multiplicative constant $m^{n / 2}$. It is standard that an atom is in $H^{1}\left(\mathbb{R}^{n}\right)$ with norm bounded by a uniform constant. The atomic decomposition gives the converse.

Theorem 2.2 (Atomic decomposition). There exists a constant $C>0$ such that all functions $f \in H^{1}\left(\mathbb{R}^{n}\right)$ can be written as the limit in the distribution sense and in $H^{1}\left(\mathbb{R}^{n}\right)$ of an infinite sum

$$
f=\sum_{j=1}^{\infty} \lambda_{j} a_{j}
$$


with $a_{j} \psi$-atoms related to some dyadic cubes $R_{j}$ and $\lambda_{j}$ constants such that

$$
C^{-1}\|f\|_{H^{1}} \leq \sum_{j=1}^{\infty}\left|\lambda_{j}\right| \leq C\|f\|_{H^{1}}
$$

This theorem is a small variation of a standard statement which can be found in [21, Section 6.5. Observe that the interest in dealing with finite atomic decompositions has been underlined recently, for instance in [34, 23].

Now, we denote by $H_{\text {fin }}^{1}\left(\mathbb{R}^{n}\right)$ the vector space of all finite linear combinations of $\psi$-atoms, that is,

$$
f=\sum_{j=1}^{k} \lambda_{j} a_{j}
$$

where $a_{j}$ 's are $\psi$-atoms. Then, the norm of $f$ in $H_{\text {fin }}^{1}\left(\mathbb{R}^{n}\right)$ is defined by

$$
\|f\|_{H_{\text {fin }}^{1}}=\inf \left\{\sum_{j=1}^{k}\left|\lambda_{j}\right|: f=\sum_{j=1}^{k} \lambda_{j} a_{j}\right\} .
$$

By the atomic decomposition theorem, the set $H_{\text {fin }}^{1}\left(\mathbb{R}^{n}\right)$ is dense in $H^{1}\left(\mathbb{R}^{n}\right)$ for the norm $\|\cdot\|_{H^{1}}$.

The following two wavelet characterizations of $L^{p}\left(\mathbb{R}^{n}\right), 1<p<\infty$, and $B M O\left(\mathbb{R}^{n}\right)$ are well known (see [35]).

Theorem 2.3. Let $1<p<\infty$. Then the norms

$$
\|f\|_{L^{p}},\left\|\left(\sum_{I} \sum_{\sigma \in E}\left|\left\langle f, \psi_{I}^{\sigma}\right\rangle\right|^{2}|I|^{-1} \chi_{I}\right)^{1 / 2}\right\|_{L^{p}} \text { and }\left\|\left(\sum_{I} \sum_{\sigma \in E}\left|\left\langle f, \psi_{I}^{\sigma}\right\rangle\right|^{2}\left(\psi_{I}^{\sigma}\right)^{2}\right)^{1 / 2}\right\|_{L^{p}}
$$

are equivalent on $L^{p}\left(\mathbb{R}^{n}\right)$.

Theorem 2.4. A function $g \in B M O\left(\mathbb{R}^{n}\right)$ if and only if

$$
\frac{1}{|R|} \sum_{I \subset R} \sum_{\sigma \in E}\left|\left\langle g, \psi_{I}^{\sigma}\right\rangle\right|^{2}<\infty
$$

for all (not necessarily dyadic) cubes $R$. Moreover, there exists a constant $C>0$ such that for all $g \in B M O\left(\mathbb{R}^{n}\right)$,

$$
C^{-1}\|g\|_{B M O} \leq \sup _{R}\left(\frac{1}{|R|} \sum_{I \subset R} \sum_{\sigma \in E}\left|\left\langle g, \psi_{I}^{\sigma}\right\rangle\right|^{2}\right)^{1 / 2} \leq C\|g\|_{B M O},
$$

where the supremum is taken over all cubes $R$.

By Theorem 2.3. Theorem 2.4 and the John-Nirenberg inequality, we obtain the following lemma. The proof is easy and will be omitted.

Lemma 2.1. Let $f$ be a $\psi$-atom related to the cube $R$ and $b \in B M O\left(\mathbb{R}^{n}\right)$. Then, $\sum_{I \subset R} \sum_{\sigma \in E}\left\langle f, \psi_{I}^{\sigma}\right\rangle\left\langle b, \psi_{I}^{\sigma}\right\rangle\left(\psi_{I}^{\sigma}\right)^{2} \in L^{q}\left(\mathbb{R}^{n}\right)$ for any $q \in(1,2)$.

\section{BilineAR, SUBBILINEAR DECOMPOSITIONS AND COMMUTATORS}

Recall that $\mathcal{K}$ is the set of all sublinear operators $T$ bounded from $H^{1}\left(\mathbb{R}^{n}\right)$ into $L^{1}\left(\mathbb{R}^{n}\right)$ satisfying

$$
\left\|\left(b-b_{Q}\right) T a\right\|_{L^{1}} \leq C\|b\|_{B M O},
$$


for all $b \in B M O\left(\mathbb{R}^{n}\right)$, any $H^{1}$-atom $a$ supported in the cube $Q$, where $C>0$ is a constant independent of $b, a$. This class $\mathcal{K}$ contains almost all important operators in harmonic analysis: Calderón-Zygmund type operators, strongly singular integral operators, multiplier operators, pseudo-differential operators, maximal type operators, the area integral operator of Lusin, Littlewood-Paley type operators, Marcinkiewicz operators, maximal Bochner-Riesz operators, etc. (see Section 4).

Here and in what follows the bilinear operator $\mathfrak{S}$ is defined by

$$
\mathfrak{S}(f, g)=-\sum_{I} \sum_{\sigma \in E}\left\langle f, \psi_{I}^{\sigma}\right\rangle\left\langle g, \psi_{I}^{\sigma}\right\rangle\left(\psi_{I}^{\sigma}\right)^{2} .
$$

In [4, the authors show that $\mathfrak{S}$ is a bounded bilinear operator from $H^{1}\left(\mathbb{R}^{n}\right) \times$ $B M O\left(\mathbb{R}^{n}\right)$ into $L^{1}\left(\mathbb{R}^{n}\right)$.

3.1. Two decomposition theorems and $\left(H_{b}^{1}, L^{1}\right)$-type estimates. Let $b$ be a locally integrable function and $T \in \mathcal{K}$. As usual, the (sublinear) commutator $[b, T]$ of the operator $T$ is defined by $[b, T](f)(x):=T((b(x)-b(\cdot)) f(\cdot))(x)$.

Theorem 3.1 (Subbilinear decomposition). Let $T \in \mathcal{K}$. There exists a bounded subbilinear operator $\mathfrak{R}=\mathfrak{R}_{T}: H^{1}\left(\mathbb{R}^{n}\right) \times B M O\left(\mathbb{R}^{n}\right) \rightarrow L^{1}\left(\mathbb{R}^{n}\right)$ such that for all $(f, b) \in H^{1}\left(\mathbb{R}^{n}\right) \times B M O\left(\mathbb{R}^{n}\right)$, we have

$$
|T(\mathfrak{S}(f, b))|-\mathfrak{R}(f, b) \leq|[b, T](f)| \leq \mathfrak{R}(f, b)+|T(\mathfrak{S}(f, b))| .
$$

Corollary 3.1. Let $T \in \mathcal{K}$ be such that $T$ is of weak type $(1,1)$. Then, the bilinear operator $\mathfrak{P}(f, g)=[g, T](f)$ maps continuously $H^{1}\left(\mathbb{R}^{n}\right) \times B M O\left(\mathbb{R}^{n}\right)$ into weak- $L^{1}\left(\mathbb{R}^{n}\right)$. In particular, the commutator $[b, T]$ is of weak type $\left(H^{1}, L^{1}\right)$ if $b \in B M O\left(\mathbb{R}^{n}\right)$.

We remark that the class of operators $T \in \mathcal{K}$ of weak type $(1,1)$ contains Calderón-Zygmund operators, strongly singular integral operators, multiplier operators, pseudo-differential operators whose symbols are in the Hörmander class $S_{\varrho, \delta}^{m}$ with $0<\varrho \leq 1,0 \leq \delta<1, m \leq-n((1-\varrho) / 2+\max \{0,(\delta-\varrho) / 2\})$, maximal type operators, the area integral operator of Lusin, Littlewood-Paley type operators, Marcinkiewicz operators, maximal Bochner-Riesz operators $T_{*}^{\delta}$ with $\delta>(n-1) / 2$, etc.

When $T$ is linear and belongs to $\mathcal{K}$, we obtain the bilinear decomposition for the linear commutator $[b, T]$ of $f,[b, T](f)=b T(f)-T(b f)$, instead of the subbilinear decomposition as stated in Theorem 3.1 .

Theorem 3.2 (Bilinear decomposition). Let $T$ be a linear operator in $\mathcal{K}$. Then, there exists a bounded bilinear operator $\mathfrak{R}=\mathfrak{R}_{T}: H^{1}\left(\mathbb{R}^{n}\right) \times B M O\left(\mathbb{R}^{n}\right) \rightarrow L^{1}\left(\mathbb{R}^{n}\right)$ such that for all $(f, b) \in H^{1}\left(\mathbb{R}^{n}\right) \times B M O\left(\mathbb{R}^{n}\right)$, we have

$$
[b, T](f)=\mathfrak{R}(f, b)+T(\mathfrak{S}(f, b)) .
$$

The following result gives $\left(H_{b}^{1}, L^{1}\right)$-type estimates for commutators $[b, T]$ when $T$ belongs to the class $\mathcal{K}$.

Theorem 3.3. Let $b$ be a non-constant BMO-function and $T \in \mathcal{K}$. Then, the commutator $[b, T]$ maps continuously $H_{b}^{1}\left(\mathbb{R}^{n}\right)$ into $L^{1}\left(\mathbb{R}^{n}\right)$. 
Note that in the particular case of $T$ a 1-Calderón-Zygmund operator and $H_{b}^{1}\left(\mathbb{R}^{n}\right)$ replaced by $\mathcal{H}_{b}^{1}\left(\mathbb{R}^{n}\right)$, Pérez 38 . proved

$$
\sup \left\{\|[b, T](a)\|_{L^{1}}: a \text { is a }(\infty, b)-\text { atom }\right\}<\infty .
$$

Then he concludes that the (linear) commutator $[b, T]$ maps continuously $\mathcal{H}_{b}^{1}\left(\mathbb{R}^{n}\right)$ into $L^{1}\left(\mathbb{R}^{n}\right)$. Notice that $\mathcal{H}_{b}^{1}\left(\mathbb{R}^{n}\right) \subset \mathcal{H}_{b}^{1, q}\left(\mathbb{R}^{n}\right) \subset H_{b}^{1}\left(\mathbb{R}^{n}\right), 1<q \leq \infty$, and the inclusions are continuous (see Section 5). However, as mentioned in the introduction, inequality (3.1) does not suffice to conclude that the (linear) commutator $[b, T]$ is bounded from $\mathcal{H}_{b}^{1}\left(\mathbb{R}^{n}\right)$ to $L^{1}\left(\mathbb{R}^{n}\right)$. We should also point out that the $\left(H^{1}, L^{1}\right)$ weak type estimates and the $\left(\mathcal{H}_{b}^{1}, L^{1}\right)$-type estimates for the (linear) commutators of multiplier operators (see [45, 25, 42]), strongly singular Calderón-Zygmund operators (see [26]) and for the (sublinear) commutators of Littlewood-Paley type operators (see [28]), Marcinkiewicz operators (see [33]), maximal Bochner-Riesz operators (see [30, 31, 29]) have been studied recently. However, the authors just prove inequality (1.2) (that is, inequality (3.1)) and use equality (1.1) which leaves a gap as pointed out in the introduction.

3.2. Boundedness of linear commutators on Hardy spaces. Analogously to Hardy estimates for bilinear operators of Coifman and Grafakos ([8]; see also [14]), we obtain the following strongly bilinear estimates which improve Corollary 3.1 .

Theorem 3.4. Let $T$ be a linear operator in $\mathcal{K}$. Assume that $A_{i}, B_{i}, i=1, \ldots, K$, are Calderón-Zygmund operators satisfying $A_{i} 1=A_{i}^{*} 1=B_{i} 1=B_{i}^{*} 1=0$, and for every $f$ and $g$ in $L^{2}\left(\mathbb{R}^{n}\right)$,

$$
\int_{\mathbb{R}^{n}}\left(\sum_{i=1}^{K} A_{i} f \cdot B_{i} g\right) d x=0 .
$$

Then, the bilinear operator $\mathfrak{T}$, defined by

$$
\mathfrak{T}(f, g)=\sum_{i=1}^{K}\left[B_{i} g, T\right]\left(A_{i} f\right),
$$

maps continuously $H^{1}\left(\mathbb{R}^{n}\right) \times B M O\left(\mathbb{R}^{n}\right)$ into $L^{1}\left(\mathbb{R}^{n}\right)$.

We now give a sufficient condition for the linear commutator $[b, T]$ to map continuously $H_{b}^{1}\left(\mathbb{R}^{n}\right)$ into $h^{1}\left(\mathbb{R}^{n}\right)$.

Theorem 3.5. Let $b$ be a non-constant BMO ${ }^{\log }$-function and $T$ be a CalderónZygmund operator with $T 1=T^{*} 1=0$. Then, the linear commutator $[b, T]$ maps continuously $H_{b}^{1}\left(\mathbb{R}^{n}\right)$ into $h^{1}\left(\mathbb{R}^{n}\right)$.

The last theorem gives a sufficient condition for the linear commutator $[b, T]$ to map continuously $H_{b}^{1}\left(\mathbb{R}^{n}\right)$ into $H^{1}\left(\mathbb{R}^{n}\right)$.

Theorem 3.6. Let $b$ be a non-constant BMO-function and $T$ be a CalderónZygmund operator with $T^{*} 1=T^{*} b=0$. Then, the linear commutator $[b, T]$ maps continuously $H_{b}^{1}\left(\mathbb{R}^{n}\right)$ into $H^{1}\left(\mathbb{R}^{n}\right)$.

Observe that the condition $T^{*} b=0$ is "necessary" in the sense that if the linear commutator $[b, T]$ maps continuously $H_{b}^{1}\left(\mathbb{R}^{n}\right)$ into $H^{1}\left(\mathbb{R}^{n}\right)$, then $\int_{\mathbb{R}^{n}} b(x) T a(x) d x=$ 0 holds for all $(q, b)$-atoms $a, 1<q \leq \infty$.

Also, let us give some examples to illustrate the sufficient conditions in Theorem 3.6. To have many examples, let us consider Euclidean spaces $\mathbb{R}^{n}, n \geq 2$. Now, 
consider all Calderón-Zygmund operators $T$ such that $T^{*} 1=0$. As the closure of $T\left(H^{1}\left(\mathbb{R}^{n}\right)\right)$ is a proper subset of $H^{1}\left(\mathbb{R}^{n}\right)$, by the Hahn-Banach theorem (note that $B M O\left(\mathbb{R}^{n}\right)$ is the dual of $\left.H^{1}\left(\mathbb{R}^{n}\right)\right)$, one may take $b$ to be a non-constant $B M O$ function such that $\int_{\mathbb{R}^{n}} b T a d x=0$ for all $H^{1}$-atoms $a$, i.e. $T^{*} b=0$, and thus $b$ and $T$ satisfy the sufficient condition in Theorem 3.6 .

\section{The CLASS $\mathcal{K}$ AND FOUR BILINEAR OPERATORS ON $H^{1}\left(\mathbb{R}^{n}\right) \times B M O\left(\mathbb{R}^{n}\right)$}

4.1. The class $\mathcal{K}$. The purpose of this subsection is to give some examples of operators in the class $\mathcal{K}$. More precisely, the class $\mathcal{K}$ contains almost all important operators in harmonic analysis: Calderón-Zygmund type operators, strongly singular integral operators, multiplier operators, pseudo-differential operators with symbols in the Hörmander class $S_{\varrho, \delta}^{m}$ with $0<\varrho \leq 1,0 \leq \delta<1, m \leq-n((1-\varrho) / 2+$ $\max \{0,(\delta-\varrho) / 2\})$ (see [2, 1]), maximal type operators, the area integral operator of Lusin, Littlewood-Paley type operators, Marcinkiewicz operators, maximal Bochner-Riesz operators $T_{*}^{\delta}$ with $\delta>(n-1) / 2$ (cf. 24]), etc. It is well known that these operators $T$ are bounded from $H^{1}\left(\mathbb{R}^{n}\right)$ into $L^{1}\left(\mathbb{R}^{n}\right)$. So, in order to establish that these are in the class $\mathcal{K}$, we just need to show that

$$
\left\|\left(b-b_{Q}\right) T a\right\|_{L^{1}} \leq C\|b\|_{B M O}
$$

for all $B M O$-functions $b$, and all $H^{1}$-atom $a$ is related to a cube $Q=Q\left[x_{0}, r\right]$ with constant $C>0$ independent of $b, a$.

Observe that the non-tangential grand maximal operator $\mathfrak{M}$ belongs to $\mathcal{K}$ since it satisfies inequality (4.1) (cf. [40]). We also refer to [20] for the (sublinear) commutators $\left[b, M_{\varphi, \alpha}\right]$ of the maximal operators $M_{\varphi, \alpha}$ (note that $M_{\varphi, 0}$ lies in $\mathcal{K}$ ).

Here we just give the proofs for Calderón-Zygmund operators (linear operators) and the area integral operator of Lusin (sublinear operator). For the other operators, we leave the proofs to the interested reader.

First recall that $P(x)=\frac{1}{\left(1+|x|^{2}\right)^{(n+1) / 2}}$ is the Poisson kernel and $u_{f}(x, t):=$ $f * P_{t}(x)$ is the Poisson integral of $f$. Then the area integral operator $S$ of Lusin is defined by

$$
S(f)(x)=\left(\int_{\Gamma(x)}\left|\nabla u_{f}(y, t)\right|^{2} t^{1-n} d y d t\right)^{1 / 2}
$$

where $\Gamma(x)$ is the cone $\left\{(y, t) \in \mathbb{R}_{+}^{n+1}:|y-x|<t\right\}$ with vertex at $x$, while $\nabla u_{f}=\left(\partial u_{f} / \partial x_{1}, \ldots, \partial u_{f} / \partial x_{1}, \partial u_{f} / \partial t\right)$ is the gradient of $u_{f}$ on $\mathbb{R}_{+}^{n+1}=\mathbb{R}^{n} \times(0, \infty)$.

Proposition 4.1. Let $\delta \in(0,1]$ and $T$ be a $\delta$-Calderón-Zygmund operator. Then $T$ satisfies inequality (4.1), and thus $T$ belongs to $\mathcal{K}$.

Proof. We cut the integral of $\left|\left(b-b_{Q}\right) T a\right|$ into two parts. By the Schwarz inequality and the boundedness of $T$ on $L^{2}\left(\mathbb{R}^{n}\right)$, we have

$$
\begin{aligned}
\int_{2 Q}\left|b(x)-b_{Q}\right||T a(x)| d x & \leq C\left(\int_{2 Q}\left|b(x)-b_{Q}\right|^{2} d x\right)^{1 / 2}\|a\|_{L^{2}} \\
& \leq C\|b\|_{B M O} ;
\end{aligned}
$$


here one used the fact that $\left|b_{2 Q}-b_{Q}\right| \leq C\|b\|_{B M O}$. Next, for $x \notin 2 Q$,

$$
\begin{aligned}
|T a(x)| & =\left|\int_{Q}\left(K(x, y)-K\left(x, x_{0}\right)\right) a(y) d y\right| \\
& \leq C \int_{Q} \frac{\left|y-x_{0}\right|^{\delta}}{\left|x-x_{0}\right|^{n+\delta}}|a(y)| d y \\
& \leq C \frac{r^{\delta}}{\left|x-x_{0}\right|^{n+\delta}} .
\end{aligned}
$$

Therefore,

$$
\int_{(2 Q)^{c}}\left|b(x)-b_{Q}\right||T a(x)| d x \leq C \int_{Q^{c}}\left|b(x)-b_{Q}\right| \frac{r^{\delta}}{\left|x-x_{0}\right|^{n+\delta}} d x \leq C\|b\|_{B M O},
$$

since the last inequality is classical (cf. 40]). This finishes the proof.

Corollary 4.1. Let $\mathcal{R}_{j}, j=1, \ldots, n$, be the classical Riesz transforms. Then, $\mathcal{R}_{j}$ belongs to $\mathcal{K}$ for all $j=1, \ldots, n$.

Proposition 4.2. The area integral operator $S$ satisfies inequality (4.1) and thus $S$ belongs to $\mathcal{K}$.

Proof. We also cut the integral of $\left|\left(b-b_{Q}\right) S(a)\right|$ into two parts. By the Schwarz inequality and the boundedness of $S$ on $L^{2}\left(\mathbb{R}^{n}\right)$, we have

$$
\begin{aligned}
\int_{2 Q}\left|b(x)-b_{Q}\right||S(a)(x)| d x & \leq C\left(\int_{2 Q}\left|b(x)-b_{Q}\right|^{2} d x\right)^{1 / 2}\|a\|_{L^{2}} \\
& \leq C\|b\|_{B M O} .
\end{aligned}
$$

Next, for $x \notin 2 Q$, by using the equality

$$
u_{a}(y, t)=\int_{\mathbb{R}^{n}} \frac{1}{t^{n}}\left(P\left(\frac{y-z}{t}\right)-P\left(\frac{y-x_{0}}{t}\right)\right) a(z) d z,
$$

since $\int_{\mathbb{R}^{n}} a(z) d z=0$, it is easy to establish that

$$
S(a)(x)=\left(\int_{\Gamma(x)}\left|\nabla u_{a}(y, t)\right|^{2} t^{1-n} d y d t\right)^{1 / 2} \leq C \frac{r}{\left|x-x_{0}\right|^{n+1}} .
$$

Therefore,

$$
\int_{(2 Q)^{c}}\left|b(x)-b_{Q}\right||S(a)(x)| d x \leq C \int_{Q^{c}}\left|b(x)-b_{Q}\right| \frac{r}{\left|x-x_{0}\right|^{n+1}} d x \leq C\|b\|_{B M O},
$$

which ends the proof.

We should point out that the Littlewood-Paley type operators can be viewed as vector-valued Calderón-Zygmund operators (see [39]). See also [20] in the context of vector-valued commutators. 
4.2. Four bilinear operators on $H^{1}\left(\mathbb{R}^{n}\right) \times B M O\left(\mathbb{R}^{n}\right)$. We now consider four bilinear operators on $H^{1}\left(\mathbb{R}^{n}\right) \times B M O\left(\mathbb{R}^{n}\right)$ which are fundamental for our bilinear decomposition theorem.

We first state some lemmas whose proofs can be found in [4].

Lemma 4.1. The bilinear operator $\Pi_{3}$ defined on $H^{1}\left(\mathbb{R}^{n}\right) \times B M O\left(\mathbb{R}^{n}\right)$ by

$$
\Pi_{3}(f, g)=\sum_{I} \sum_{\sigma \in E}\left\langle f, \psi_{I}^{\sigma}\right\rangle\left\langle g, \psi_{I}^{\sigma}\right\rangle\left(\psi_{I}^{\sigma}\right)^{2}
$$

is a bounded bilinear operator from $H^{1}\left(\mathbb{R}^{n}\right) \times B M O\left(\mathbb{R}^{n}\right)$ into $L^{1}\left(\mathbb{R}^{n}\right)$.

Observe that $\mathfrak{S}(f, g)=-\Pi_{3}(f, g)$ for all $(f, g) \in H^{1}\left(\mathbb{R}^{n}\right) \times B M O\left(\mathbb{R}^{n}\right)$.

Lemma 4.2. The bilinear operator $\Pi_{4}$, defined on $H^{1}\left(\mathbb{R}^{n}\right) \times B M O\left(\mathbb{R}^{n}\right)$ by

$$
\Pi_{4}(f, g)=\sum_{I, I^{\prime}} \sum_{\sigma, \sigma^{\prime} \in E}\left\langle f, \psi_{I}^{\sigma}\right\rangle\left\langle g, \psi_{I^{\prime}}^{\sigma^{\prime}}\right\rangle \psi_{I}^{\sigma} \psi_{I^{\prime}}^{\sigma^{\prime}},
$$

the sums being taken over all dyadic cubes $I, I^{\prime}$ and $\sigma, \sigma^{\prime} \in E$ such that $(I, \sigma) \neq$ $\left(I^{\prime}, \sigma^{\prime}\right)$, is a bounded bilinear operator from $H^{1}\left(\mathbb{R}^{n}\right) \times B M O\left(\mathbb{R}^{n}\right)$ into $H^{1}\left(\mathbb{R}^{n}\right)$.

Lemma 4.3. The bilinear operator $\Pi_{1}$ defined by

$$
\Pi_{1}(a, g)=\sum_{|I|=\left|I^{\prime}\right|} \sum_{\sigma \in E}\left\langle a, \phi_{I}\right\rangle\left\langle g, \psi_{I^{\prime}}^{\sigma}\right\rangle \phi_{I} \psi_{I^{\prime}}^{\sigma},
$$

where $a$ is a $\psi$-atom and $g \in B M O\left(\mathbb{R}^{n}\right)$, can be extended into a bounded bilinear operator from $H^{1}\left(\mathbb{R}^{n}\right) \times B M O\left(\mathbb{R}^{n}\right)$ into $H^{1}\left(\mathbb{R}^{n}\right)$.

Lemma 4.4. The bilinear operator $\Pi_{2}$ defined by

$$
\Pi_{2}(a, g)=\sum_{|I|=\left|I^{\prime}\right|} \sum_{\sigma \in E}\left\langle a, \psi_{I}^{\sigma}\right\rangle\left\langle g, \phi_{I^{\prime}}\right\rangle \psi_{I}^{\sigma} \phi_{I^{\prime}},
$$

where $a$ is a $\psi$-atom related to the cube $R$ and $g \in B M O\left(\mathbb{R}^{n}\right)$ can be extended into a bounded bilinear operator from $H^{1}\left(\mathbb{R}^{n}\right) \times B M O^{+}\left(\mathbb{R}^{n}\right)$ into $H^{\log }\left(\mathbb{R}^{n}\right)$. Furthermore, we can write

$$
\Pi_{2}(a, g)=h^{(1)}+\kappa g_{R} h^{(2)},
$$

where $\left\|h^{(1)}\right\|_{H^{1}} \leq C\|g\|_{B M O}, h^{(2)}$ is an atom related to $m R$, and $\kappa$ is a uniform constant, independent of $a$ and $g$.

The following remarks are useful in our proofs in Section 6 and Section 7.

Remark 4.1

(1) If $g \in B M O\left(\mathbb{R}^{n}\right)$ and $f \in H^{1}\left(\mathbb{R}^{n}\right)$ such that $f g \in L^{1}\left(\mathbb{R}^{n}\right)$, then

$$
\int_{\mathbb{R}^{n}} f g d x=\int_{\mathbb{R}^{n}} \mathfrak{S}(f, g) d x=\sum_{I} \sum_{\sigma \in E}\left\langle f, \psi_{I}^{\sigma}\right\rangle\left\langle g, \psi_{I}^{\sigma}\right\rangle .
$$

(2) For any $(f, g) \in H^{1}\left(\mathbb{R}^{n}\right) \times B M O\left(\mathbb{R}^{n}\right)$ and $c$ a constant, we have

$$
\Pi_{i}(f, g)=\Pi_{i}(f, g+c), i=1,3,4 .
$$

(3) As a consequence of Lemma 4.4 if $g_{R}=0$, then equality (4.2) gives that $\Pi_{2}(a, g) \in H^{1}\left(\mathbb{R}^{n}\right)$. Moreover, $\left\|\Pi_{2}(a, g)\right\|_{H^{1}} \leq C\|g\|_{B M O}$.

In 4], the authors have shown the following decomposition theorem for the product space $H^{1}\left(\mathbb{R}^{n}\right) \times B M O\left(\mathbb{R}^{n}\right)$. 
Theorem 4.1 (Decomposition theorem). Let $f \in H^{1}\left(\mathbb{R}^{n}\right)$ and $g \in B M O\left(\mathbb{R}^{n}\right)$. Then, we have the following decomposition:

$$
f g=\Pi_{1}(f, g)+\Pi_{2}(f, g)+\Pi_{3}(f, g)+\Pi_{4}(f, g),
$$

that is

$$
\begin{gathered}
f g=\Pi_{1}(f, g)+\Pi_{2}(f, g)+\Pi_{4}(f, g)-\mathfrak{S}(f, g) . \\
\text { 5. THe SPACE } H_{b}^{1}\left(\mathbb{R}^{n}\right)
\end{gathered}
$$

Let $b$ be a non-constant $B M O$-function. In this section, we study the space $H_{b}^{1}\left(\mathbb{R}^{n}\right)$. In particular, we give some characterizations of the space $H_{b}^{1}\left(\mathbb{R}^{n}\right)$ (see Theorem 5.1), and the comparison with the space $\mathcal{H}_{b}^{1}\left(\mathbb{R}^{n}\right)$ of Pérez (see Theorem 5.2 .

First, let us consider the class $\widetilde{\mathcal{K}}$ of all $T \in \mathcal{K}$ such that $T$ characterizes the space $H^{1}\left(\mathbb{R}^{n}\right)$, which means that $f \in H^{1}\left(\mathbb{R}^{n}\right)$ if and only if $T f \in L^{1}\left(\mathbb{R}^{n}\right)$. Clearly, the class $\widetilde{\mathcal{K}}$ contain the maximal operator $\mathfrak{M}$, the area integral operator $S$ of Lusin, the Littlewood-Paley $g$-operator (see [15]), the Littlewood-Paley $g_{\lambda}^{*}$-operator with $\lambda>3 n$ (see [19]), etc.

Here and in what follows, the symbol $f \approx g$ means that $C^{-1} f \leq g \leq C f$ for some constant $C>0$. We obtain the following characterization of $H_{b}^{1}\left(\mathbb{R}^{n}\right)$.

Theorem 5.1. Let $b$ be a non-constant BMO-function and $T \in \widetilde{\mathcal{K}}$. For $f \in$ $H^{1}\left(\mathbb{R}^{n}\right)$, the following conditions are equivalent:

i) $f \in H_{b}^{1}\left(\mathbb{R}^{n}\right)$.

ii) $\mathfrak{S}(f, b) \in H^{1}\left(\mathbb{R}^{n}\right)$.

iii) $\left[b, \mathcal{R}_{j}\right](f) \in L^{1}\left(\mathbb{R}^{n}\right)$ for all $j=1, \ldots, n$.

iv) $[b, T](f) \in L^{1}\left(\mathbb{R}^{n}\right)$.

Furthermore, if one of these conditions is satisfied, then

$$
\begin{aligned}
\|f\|_{H_{b}^{1}} & =\|f\|_{H^{1}}\|b\|_{B M O}+\|[b, \mathfrak{M}](f)\|_{L^{1}} \\
& \approx\|f\|_{H^{1}}\|b\|_{B M O}+\|\mathfrak{S}(f, b)\|_{H^{1}} \\
& \approx\|f\|_{H^{1}}\|b\|_{B M O}+\sum_{j=1}^{n}\left\|\left[b, \mathcal{R}_{j}\right](f)\right\|_{L^{1}} \\
& \approx\|f\|_{H^{1}}\|b\|_{B M O}+\|[b, T](f)\|_{L^{1}},
\end{aligned}
$$

where the constants are independent of $f$ and $b$.

Remark 5.1. Theorem 3.3 and Theorem 5.1 give that $[b, T]$ is bounded from $H_{b}^{1}\left(\mathbb{R}^{n}\right)$ to $L^{1}\left(\mathbb{R}^{n}\right)$ for every $T$ a Calderón-Zygmund singular integral operator. Furthermore, $H_{b}^{1}\left(\mathbb{R}^{n}\right)$ is the largest space having this property.

Proof of Theorem 5.1. $(i) \Leftrightarrow(i i)$ By Theorem 3.1, there exists a bounded subbilinear operator $\mathfrak{R}: H^{1}\left(\mathbb{R}^{n}\right) \times B M O\left(\mathbb{R}^{n}\right) \rightarrow L^{1}\left(\mathbb{R}^{n}\right)$ such that

$$
\mathfrak{M}(\mathfrak{S}(f, b))-\mathfrak{R}(f, b) \leq|[b, \mathfrak{M}](f)| \leq \mathfrak{R}(f, b)+\mathfrak{M}(\mathfrak{S}(f, b)) .
$$

Consequently, $\mathfrak{S}(f, b) \in H^{1}\left(\mathbb{R}^{n}\right)$ if and only if $[b, \mathfrak{M}](f) \in L^{1}\left(\mathbb{R}^{n}\right)$. Moreover,

$$
\|f\|_{H_{b}^{1}} \approx\|f\|_{H^{1}}\|b\|_{B M O}+\|\mathfrak{S}(f, b)\|_{H^{1}} .
$$

(ii) $\Leftrightarrow($ iii $)$. By Theorem 3.2 , there exist $n$ bounded bilinear operators $\mathfrak{R}_{j}$ : $H^{1}\left(\mathbb{R}^{n}\right) \times B M O\left(\mathbb{R}^{n}\right) \rightarrow L^{1}\left(\mathbb{R}^{n}\right), j=1, \ldots, n$, such that

$$
\left[b, \mathcal{R}_{j}\right](f)=\mathfrak{R}_{j}(f, b)+\mathcal{R}_{j}(\mathfrak{S}(f, b)) .
$$


Consequently, $\mathfrak{S}(f, b) \in H^{1}\left(\mathbb{R}^{n}\right)$ if and only if $\left[b, \mathcal{R}_{j}\right](f) \in L^{1}\left(\mathbb{R}^{n}\right)$ for all $j=1, \ldots, n$. Moreover,

$$
\|f\|_{H^{1}}\|b\|_{B M O}+\|\mathfrak{S}(f, b)\|_{H^{1}} \approx\|f\|_{H^{1}}\|b\|_{B M O}+\sum_{j=1}^{n}\left\|\left[b, \mathcal{R}_{j}\right](f)\right\|_{L^{1}} .
$$

(ii) $\Leftrightarrow($ iv). By Theorem 3.1 there exists a bounded subbilinear operator $\mathfrak{R}$ : $H^{1}\left(\mathbb{R}^{n}\right) \times B M O\left(\mathbb{R}^{n}\right) \rightarrow L^{1}\left(\mathbb{R}^{n}\right)$ such that

$$
|T(\mathfrak{S}(f, b))|-\mathfrak{R}(f, b) \leq|[b, T](f)| \leq \mathfrak{R}(f, b)+|T(\mathfrak{S}(f, b))| .
$$

Consequently, $\mathfrak{S}(f, b) \in H^{1}\left(\mathbb{R}^{n}\right)$ if and only if $[b, T](f) \in L^{1}\left(\mathbb{R}^{n}\right)$ since $T \in \widetilde{\mathcal{K}}$. Moreover,

$$
\|f\|_{H^{1}}\|b\|_{B M O}+\|\mathfrak{S}(f, b)\|_{H^{1}} \approx\|f\|_{H^{1}}\|b\|_{B M O}+\|[b, T](f)\|_{L^{1}} .
$$

Note that the constants in the last equivalence depend on $T$.

The following lemma is an immediate corollary of the weak convergence theorem in $H^{1}\left(\mathbb{R}^{n}\right)$ of Jones and Journé. See also [1] in the setting of $h^{1}\left(\mathbb{R}^{n}\right)$.

Lemma 5.1. Let $\left\{f_{k}\right\}_{k \geq 1}$ be a bounded sequence in $H^{1}\left(\mathbb{R}^{n}\right)$ (resp., in $h^{1}\left(\mathbb{R}^{n}\right)$ ) such that $f_{k}$ tends to $f$ in $L^{1}\left(\mathbb{R}^{n}\right)$. Then $f$ in $H^{1}\left(\mathbb{R}^{n}\right)$ (resp., in $\left.h^{1}\left(\mathbb{R}^{n}\right)\right)$, and

$$
\|f\|_{H^{1}} \leq \varliminf_{k \rightarrow \infty}\left\|f_{k}\right\|_{H^{1}} \quad\left(\text { resp. },\|f\|_{h^{1}} \leq \varliminf_{k \rightarrow \infty}\left\|f_{k}\right\|_{h^{1}}\right) \text {. }
$$

Theorem 5.2. Let $b$ be a non-constant BMO-function and $1<q \leq \infty$. Then, $\mathcal{H}_{b}^{1, q}\left(\mathbb{R}^{n}\right) \subset H_{b}^{1}\left(\mathbb{R}^{n}\right)$ and the inclusion is continuous.

Proof. Let $a$ be a $(q, b)$-atom related to the cube $Q$. We first prove that $\left(b-b_{Q}\right) a$ is $C\|b\|_{B M O}$ times a classical $(\widetilde{q}+1) / 2$-atom. One has supp $\left(b-b_{Q}\right) a \subset \operatorname{supp} a \subset Q$ and $\int_{\mathbb{R}^{n}}\left(b(x)-b_{Q}\right) a(x) d x=\int_{\mathbb{R}^{n}} b(x) a(x) d x-b_{Q} \int_{\mathbb{R}^{n}} a(x) d x=0$. Moreover, by the Hölder inequality and the John-Nirenberg inequality, we get

$$
\left\|\left(b-b_{Q}\right) a\right\|_{L^{(\widetilde{q}+1) / 2}} \leq\left\|\left(b-b_{Q}\right) \chi_{Q}\right\|_{L^{\tilde{q}(\widetilde{q}+1) /(\widetilde{q}-1)}}\|a\|_{L^{\widetilde{q}}} \leq C\|b\|_{B M O}|Q|^{(-\widetilde{q}+1) /(\widetilde{q}+1)},
$$

where $\widetilde{q}=q$ if $1<q<\infty, \widetilde{q}=2$ if $q=\infty$, and $C>0$ is independent of $b, a$. Hence, $\left(b-b_{Q}\right) a$ is $C\|b\|_{B M O}$ times a classical $(\widetilde{q}+1) / 2$-atom, and $\left\|\left(b-b_{Q}\right) a\right\|_{H^{1}} \leq$ $C\|b\|_{B M O}$.

We now prove that $\mathfrak{S}(a, b)$ belongs to $H^{1}$.

By Theorem 3.2, there exist $n$ bounded bilinear operators $\mathfrak{R}_{j}: H^{1}\left(\mathbb{R}^{n}\right) \times$ $B M O\left(\mathbb{R}^{n}\right) \rightarrow L^{1}\left(\mathbb{R}^{n}\right), j=1, \ldots, n$, such that

$$
\left[b, \mathcal{R}_{j}\right](a)=\mathfrak{R}_{j}(a, b)+\mathcal{R}_{j}(\mathfrak{S}(a, b)),
$$

since $\mathcal{R}_{j}$ is linear and belongs to $\mathcal{K}$ (see Corollary 4.1). Consequently, for all $j=1, \ldots, n$, as $\mathcal{R}_{j} \in \mathcal{K}$,

$$
\begin{aligned}
\left\|\mathcal{R}_{j}(\mathfrak{S}(a, b))\right\|_{L^{1}}= & \left\|\left(b-b_{Q}\right) \mathcal{R}_{j}(a)-\mathcal{R}_{j}\left(\left(b-b_{Q}\right) a\right)-\mathfrak{R}_{j}(a, b)\right\|_{L^{1}} \\
\leq & \left\|\left(b-b_{Q}\right) \mathcal{R}_{j}(a)\right\|_{L^{1}}+\left\|\mathcal{R}_{j}\right\|_{H^{1} \rightarrow L^{1}}\left\|\left(\left(b-b_{Q}\right) a\right)\right\|_{H^{1}} \\
& +\left\|\mathfrak{R}_{j}(a, b)\right\|_{L^{1}} \\
\leq & C\|b\|_{B M O} .
\end{aligned}
$$

This proves that $\mathfrak{S}(a, b) \in H^{1}\left(\mathbb{R}^{n}\right)$ since $\|\mathfrak{S}(a, b)\|_{L^{1}} \leq C\|b\|_{B M O}$, and moreover that

$$
\|\mathfrak{S}(a, b)\|_{H^{1}} \leq C\|b\|_{B M O} .
$$


Now, for any $f \in \mathcal{H}_{b}^{1, q}\left(\mathbb{R}^{n}\right)$, there exists an expansion $f=\sum_{j=1}^{\infty} \lambda_{j} a_{j}$, where the $a_{j}$ 's are $(q, b)$-atoms and $\sum_{j=1}^{\infty}\left|\lambda_{j}\right| \leq 2\|f\|_{\mathcal{H}_{b}^{1, q}}$. Then the sequence $\left\{\sum_{j=1}^{k} \lambda_{j} a_{j}\right\}_{k \geq 1}$ converges to $f$ in $\mathcal{H}_{b}^{1, q}\left(\mathbb{R}^{n}\right)$ and thus in $H^{1}\left(\mathbb{R}^{n}\right)$. Hence, Lemma 4.1 implies that the sequence $\left\{\mathfrak{S}\left(\sum_{j=1}^{k} \lambda_{j} a_{j}, b\right)\right\}_{k \geq 1}$ converges to $\mathfrak{S}(f, b)$ in $L^{1}\left(\mathbb{R}^{n}\right)$. In addition, by (5.1),

$$
\left\|\mathfrak{S}\left(\sum_{j=1}^{k} \lambda_{j} a_{j}, b\right)\right\|_{H^{1}} \leq \sum_{j=1}^{k}\left|\lambda_{j}\right|\left\|\mathfrak{S}\left(a_{j}, b\right)\right\|_{H^{1}} \leq C\|f\|_{\mathcal{H}_{b}^{1, q}}\|b\|_{B M O} .
$$

We then use Lemma 5.1 to conclude that $\mathfrak{S}(f, b) \in H^{1}\left(\mathbb{R}^{n}\right)$, and thus $f \in H_{b}^{1}\left(\mathbb{R}^{n}\right)$ (see Theorem 5.1). Moreover,

$$
\begin{aligned}
\|f\|_{H_{b}^{1}} & \leq C\left(\|f\|_{H^{1}}\|b\|_{B M O}+\|\mathfrak{S}(f, b)\|_{H^{1}}\right) \\
& \leq C\left(\|f\|_{\mathcal{H}_{b}^{1, q}}\|b\|_{B M O}+\underline{\lim }_{k \rightarrow \infty}\left\|\mathfrak{S}\left(\sum_{j=1}^{k} \lambda_{j} a_{j}, b\right)\right\|_{H^{1}}\right) \\
& \leq C\|f\|_{\mathcal{H}_{b}^{1, q}}\|b\|_{B M O},
\end{aligned}
$$

which ends the proof.

From Theorem 3.3 and Theorem [5.1, we get the following corollary.

Corollary 5.1. Let $b$ be a BMO-function, $T \in \mathcal{K}$ and $1<q \leq \infty$. Then the linear commutator $[b, T]$ maps continuously $\mathcal{H}_{b}^{1, q}\left(\mathbb{R}^{n}\right)$ into $L^{1}\left(\mathbb{R}^{n}\right)$.

\section{Proof of Theorem 3.1, Theorem 3.2, Theorem 3.3}

In order to prove the decomposition theorems (Theorem 3.2 and Theorem 3.1 ), we need the following two lemmas.

Lemma 6.1. Let $T \in \mathcal{K}$ and a be a classical $H^{1}$-atom related to the cube $m Q$. Then, there exists a positive constant $C=C(m)$ such that

$$
\left\|\left(g-g_{Q}\right) T a\right\|_{L^{1}} \leq C\|g\|_{B M O}, \text { for all } g \in B M O\left(\mathbb{R}^{n}\right) .
$$

Proof. Since $T \in \mathcal{K}$ and since $\left|g_{Q}-g_{m Q}\right| \leq C(m)\|g\|_{B M O}$, we have

$$
\left\|\left(g-g_{Q}\right) T a\right\|_{L^{1}} \leq C(m)\|g\|_{B M O}\|T a\|_{L^{1}}+\left\|\left(g-g_{m Q}\right) T a\right\|_{L^{1}} \leq C\|g\|_{B M O} .
$$

Lemma 6.2. The norms $\|\cdot\|_{H^{1}}$ and $\|\cdot\|_{H_{\text {fin }}^{1}}$ are equivalent on $H_{\mathrm{fin}}^{1}\left(\mathbb{R}^{n}\right)$.

We point out that in the proof below we use the results and notation of Theorem 5.12 of [21]. Even though the proofs in [21] are in the one-dimensional case, they can be easily carried out in higher dimensions as well.

The proof of Lemma 6.2. Obviously, $H_{\text {fin }}^{1}\left(\mathbb{R}^{n}\right) \subset H^{1}\left(\mathbb{R}^{n}\right)$ and for all $f \in H_{\text {fin }}^{1}\left(\mathbb{R}^{n}\right)$, we have $\|f\|_{H^{1}} \leq C\|f\|_{H_{\text {fin }}^{1}}$. We now have to show that there exists a constant $C>0$ such that for all $f \in H_{\text {fin }}^{1}\left(\mathbb{R}^{n}\right)$,

$$
\|f\|_{H_{\text {fin }}^{1}} \leq C\|f\|_{H^{1}} .
$$


By homogeneity, we can assume that $\|f\|_{H^{1}}=1$. We write $f=\sum_{j=1}^{N_{0}} \lambda_{j} a_{j}$, where the $a_{j}$ 's are $\psi$-atoms related to the cubes $R_{j}$. Since $f \in L^{2}\left(\mathbb{R}^{n}\right) \cap H^{1}\left(\mathbb{R}^{n}\right)$, there exists a $\psi$-atomic decomposition (see 21, Theorem 5.12)

$$
f=\sum_{I} \sum_{\sigma \in E}\left\langle f, \psi_{I}^{\sigma}\right\rangle \psi_{I}^{\sigma}=\sum_{k \in \mathbb{Z}} \sum_{i \in \Lambda_{k}}\left(\sum_{I \subset \widetilde{I}_{k}^{i}, I \in \mathcal{B}_{k}} \sum_{\sigma \in E}\left\langle f, \psi_{I}^{\sigma}\right\rangle \psi_{I}^{\sigma}\right),
$$

where $\sum_{I \subset \widetilde{I}_{k}^{i}, I \in \mathcal{B}_{k}} \sum_{\sigma \in E}\left\langle f, \psi_{I}^{\sigma}\right\rangle \psi_{I}^{\sigma}=\lambda(k, i) a_{k, i}$ with $a_{k, i} \psi$-atoms related to the cubes $m \widetilde{I}_{k}^{i}$ and

$$
\sum_{k \in \mathbb{Z}} \sum_{i \in \Lambda_{k}}|\lambda(k, i)| \leq C\|f\|_{H^{1}}=C .
$$

We note that $\operatorname{supp} a_{k, i} \subset \bigcup_{j=1}^{N_{0}} m R_{j}$ for all $k \in \mathbb{Z}, i \in \Lambda_{k}$. Recall that

$$
\begin{aligned}
\mathcal{W}_{\psi} f & =\left(\sum_{I} \sum_{\sigma \in E}\left|\left\langle f, \psi_{I}^{\sigma}\right\rangle\right|^{2}|I|^{-1} \chi_{I}\right)^{1 / 2} \\
& =\left(\sum_{j=1}^{N_{0}} \sum_{I \subset R_{j}} \sum_{\sigma \in E}\left|\left\langle f, \psi_{I}^{\sigma}\right\rangle\right|^{2}|I|^{-1} \chi_{I}\right)^{1 / 2}
\end{aligned}
$$

and $\Omega_{k}=\left\{x \in \mathbb{R}^{n}: \mathcal{W}_{\psi} f(x)>2^{k}\right\}$ for any $k \in \mathbb{Z}$. Clearly, supp $\mathcal{W}_{\psi} f \subset \bigcup_{j=1}^{N_{0}} m R_{j}$. So, there exists a cube $Q$ such that $\Omega_{k} \subset \operatorname{supp} \mathcal{W}_{\psi} f \subset \bigcup_{j=1}^{N_{0}} m R_{j} \subset Q$ for all $k \in \mathbb{Z}$. We now denote by $k^{\prime}$ the largest integer $k$ such that $2^{k} \leq|Q|^{-1}$. Then, we define the functions $g$ and $\ell$ by

$$
g=\sum_{k \leq k^{\prime}} \sum_{i \in \Lambda_{k}}\left(\sum_{I \subset \widetilde{I}_{k}^{i}, I \in \mathcal{B}_{k}} \sum_{\sigma \in E}\left\langle f, \psi_{I}^{\sigma}\right\rangle \psi_{I}^{\sigma}\right)
$$

and

$$
\ell=\sum_{k>k^{\prime}} \sum_{i \in \Lambda_{k}}\left(\sum_{I \subset \widetilde{I}_{k}^{i}, I \in \mathcal{B}_{k}} \sum_{\sigma \in E}\left\langle f, \psi_{I}^{\sigma}\right\rangle \psi_{I}^{\sigma}\right) .
$$

Obviously, $f=g+\ell$; moreover, supp $g \subset Q$ and supp $\ell \subset Q$. On the other hand, it follows from Theorem 5.12 of 21] that $\sum_{I \subset \widetilde{I}_{k}^{i}, I \in \mathcal{B}_{k}} \sum_{\sigma \in E}\left|\left\langle f, \psi_{I}^{\sigma}\right\rangle\right|^{2} \leq$ $C 2^{2 k}\left|\widetilde{I}_{k}^{i} \cap \Omega_{k}\right|$. Hence, as the dyadic cubes $\widetilde{I}_{k}^{i}$ are disjoint (see also [21]), we get

$$
\begin{aligned}
\|g\|_{L^{2}}^{2} & \leq C \sum_{k \leq k^{\prime}} \sum_{i \in \Lambda_{k}} \sum_{I \subset \widetilde{I}_{k}^{i}, I \in \mathcal{B}_{k}} \sum_{\sigma \in E}\left|\left\langle f, \psi_{I}^{\sigma}\right\rangle\right|^{2} \\
& \leq C \sum_{k \leq k^{\prime}} \sum_{i \in \Lambda_{k}} 2^{2 k}\left|\widetilde{I}_{k}^{i} \cap \Omega_{k}\right| \leq C \sum_{k \leq k^{\prime}} 2^{2 k}\left|\Omega_{k}\right| \\
& \leq C 2^{2 k^{\prime}}|Q| \leq C|Q|^{-1} .
\end{aligned}
$$

This proves that $C^{-1 / 2} g$ is a $\psi$-atom related to the cube $Q$.

Now, for any positive integer $K$, set $F_{K}=\left\{(k, i): k>k^{\prime},|k|+|i| \leq K\right\}$ and $\ell_{K}=\sum_{(k, i) \in F_{K}}\left(\sum_{I \subset \widetilde{I}_{k}^{i}, I \in \mathcal{B}_{k}} \sum_{\sigma \in E}\left\langle f, \psi_{I}^{\sigma}\right\rangle \psi_{I}^{\sigma}\right)$. Observe that since $f \in L^{2}\left(\mathbb{R}^{n}\right)$, the series $\sum_{k>k^{\prime}} \sum_{i \in \Lambda_{k}}\left(\sum_{I \subset \widetilde{I}_{k}^{i}, I \in \mathcal{B}_{k}} \sum_{\sigma \in E}\left\langle f, \psi_{I}^{\sigma}\right\rangle \psi_{I}^{\sigma}\right)$ converges in $L^{2}\left(\mathbb{R}^{n}\right)$. So, for any $\varepsilon>0$, if $K$ is large enough, $\varepsilon^{-1}\left(\ell-\ell_{K}\right)$ is a $\psi$-atom related to the cube $Q$. 
Therefore, $f=g+\ell_{K}+\left(\ell-\ell_{K}\right)$ is a finite linear combination of atoms for $f$, and thus

$$
\begin{aligned}
\|f\|_{H_{\mathrm{fin}}^{1}} & \leq C\left(\|g\|_{H_{\mathrm{fin}}^{1}}+\left\|\ell_{K}\right\|_{H_{\mathrm{fin}}^{1}}+\left\|\ell-\ell_{K}\right\|_{H_{\mathrm{fin}}^{1}}\right) \\
& \leq C\left(C+\sum_{k \in \mathbb{Z}} \sum_{i \in \Lambda_{k}}|\lambda(k, i)|+\varepsilon\right) \leq C
\end{aligned}
$$

by (6.1). This ends the proof.

Proof of Theorem 3.1 . We define the subbilinear operator $\mathfrak{R}$ by

$$
\mathfrak{R}(f, b)(x):=\left|T\left(b(x) f(\cdot)-\Pi_{2}(f, b)(\cdot)\right)(x)\right|+\left|T\left(\Pi_{1}(f, b)\right)(x)\right|+\left|T\left(\Pi_{4}(f, b)\right)(x)\right|
$$

for all $(f, b) \in H^{1}\left(\mathbb{R}^{n}\right) \times B M O\left(\mathbb{R}^{n}\right)$. Then, by Theorem 4.1, we obtain that

$$
|T(\mathfrak{S}(f, b))|-\mathfrak{R}(f, b) \leq|[b, T](f)| \leq \mathfrak{R}(f, b)+|T(\mathfrak{S}(f, b))| .
$$

By Lemma 4.1. Lemma 4.2 and Lemma 4.3, it is sufficient to show that the subbilinear operator

$$
\mathfrak{U}(f, b)(x):=\left|T\left(b(x) f(\cdot)-\Pi_{2}(f, b)(\cdot)\right)(x)\right|
$$

is bounded from $H^{1}\left(\mathbb{R}^{n}\right) \times B M O\left(\mathbb{R}^{n}\right)$ into $L^{1}\left(\mathbb{R}^{n}\right)$.

We first consider $b \in B M O\left(\mathbb{R}^{n}\right)$ and $f$ a $\psi$-atom related to the cube $Q$. Then, by Remark 4.1, we have

$$
\mathfrak{U}(f, b)(x)=\mathfrak{U}\left(f, b-b_{Q}\right)(x) \leq\left|\left(b(x)-b_{Q}\right) T f(x)\right|+\left|T\left(\Pi_{2}\left(f, b-b_{Q}\right)\right)(x)\right| .
$$

Consequently, by Remark 4.1, Lemma 6.1 and the fact that $f$ is $C$ times a classical atom related to the cube $m Q$, we obtain that

$$
\|\mathfrak{U}(f, b)\|_{L^{1}} \leq\left\|\left(b-b_{Q}\right) T f\right\|_{L^{1}}+\|T\|_{H^{1} \rightarrow L^{1}}\left\|\Pi_{2}\left(f, b-b_{Q}\right)\right\|_{H^{1}} \leq C\|b\|_{B M O},
$$

where $C>0$ is independent of $f, b$.

Now, let $b \in B M O\left(\mathbb{R}^{n}\right)$ and $f \in H_{\text {fin }}^{1}\left(\mathbb{R}^{n}\right)$. By Lemma 6.2 there exists a finite decomposition $f=\sum_{j=1}^{k} \lambda_{j} a_{j}$ such that $\sum_{j=1}^{k}\left|\lambda_{j}\right| \leq C\|f\|_{H^{1}}$. Consequently, by (6.2), we obtain that

$$
\|\mathfrak{U}(f, b)\|_{L^{1}} \leq \sum_{j=1}^{k}\left|\lambda_{j}\right|\left\|\mathfrak{U}\left(a_{j}, b\right)\right\|_{L^{1}} \leq C\|f\|_{H^{1}}\|b\|_{B M O},
$$

which ends the proof as $H_{\text {fin }}^{1}\left(\mathbb{R}^{n}\right)$ is dense in $H^{1}\left(\mathbb{R}^{n}\right)$ for the norm $\|\cdot\|_{H^{1}}$.

Proof of Theorem 3.2. We define the bilinear operator $\mathfrak{R}$ by

$$
\mathfrak{R}(f, b)=\left(b T f-T\left(\Pi_{2}(f, b)\right)\right)-T\left(\Pi_{1}(f, b)+\Pi_{4}(f, b)\right),
$$

for all $(f, b) \in H^{1}\left(\mathbb{R}^{n}\right) \times B M O\left(\mathbb{R}^{n}\right)$. Then, it follows from Theorem 4.1 and the proof of Theorem 3.1 that

$$
[b, T](f)=\mathfrak{R}(f, b)+T(\mathfrak{S}(f, b)),
$$

where the bilinear operator $\mathfrak{R}$ is bounded from $H^{1}\left(\mathbb{R}^{n}\right) \times B M O\left(\mathbb{R}^{n}\right)$ into $L^{1}\left(\mathbb{R}^{n}\right)$. This completes the proof.

Proof of Theorem 3.3. Theorem 3.3 is an immediate corollary of Theorem 3.1 and Theorem 5.1 


\section{Proof of Theorem 3.4, Theorem 3.5 and Theorem 3.6}

First we recall the following well-known result.

Theorem A (see [8] or [14]). Let $T$ be a Calderón-Zygmund operator satisfying $T 1=T^{*} 1=0,1<q<\infty$ and $1 / p+1 / q=1$. Then, fTg $-g T^{*} f \in H^{1}\left(\mathbb{R}^{n}\right)$ for all $f \in L^{p}\left(\mathbb{R}^{n}\right), g \in L^{q}\left(\mathbb{R}^{n}\right)$.

Now, in order to prove the bilinear type estimates and the Hardy type theorems for the commutators of Calderón-Zygmund operators, we need the following three technical lemmas.

Lemma 7.1. Let $\delta \in(0,1]$, and $A, B$ be two $\delta$-Calderón-Zygmund operators such that $A 1=A^{*} 1=B 1=B^{*} 1=0$. Then, there exists a constant $C=C(n, \delta)$ such that

$$
\sum_{I, I^{\prime}, I^{\prime \prime}} \sum_{\sigma, \sigma^{\prime}, \sigma^{\prime \prime} \in E}\left|\left\langle f, \psi_{I}^{\sigma}\right\rangle\left\langle g, \psi_{I^{\prime}}^{\sigma^{\prime}}\right\rangle\left\langle A \psi_{I}^{\sigma}, \psi_{I^{\prime \prime}}^{\sigma^{\prime \prime}}\right\rangle\left\langle B \psi_{I^{\prime}}^{\sigma^{\prime}}, \psi_{I^{\prime \prime}}^{\sigma^{\prime \prime}}\right\rangle\right| \leq C\|f\|_{H^{1}}\|g\|_{B M O}
$$

for all $f \in H^{1}\left(\mathbb{R}^{n}\right), g \in B M O\left(\mathbb{R}^{n}\right)$.

Lemma 7.2. Let $\delta \in(0,1]$, and $A_{i}, B_{i}, i=1, \ldots, K$, be $\delta$-Calderón-Zygmund operators satisfying $A_{i} 1=A_{i}^{*} 1=B_{i} 1=B_{i}^{*} 1=0$, and for every $f$ and $g$ in $L^{2}\left(\mathbb{R}^{n}\right)$,

$$
\int_{\mathbb{R}^{n}}\left(\sum_{i=1}^{K} A_{i} f \cdot B_{i} g\right) d x=0 .
$$

Then, the bilinear operator $\mathfrak{P}$, defined by $\mathfrak{P}(f, g)=\sum_{i=1}^{K} \mathfrak{S}\left(A_{i} f, B_{i} g\right)$, maps continuously $H^{1}\left(\mathbb{R}^{n}\right) \times B M O\left(\mathbb{R}^{n}\right)$ into $H^{1}\left(\mathbb{R}^{n}\right)$.

Corollary 7.1. Let $T$ be a Calderón-Zygmund operator satisfying $T 1=T^{*} 1=0$. Then the bilinear operator $\mathfrak{P}$, defined by $\mathfrak{P}(f, g)=\mathfrak{S}(T f, g)-\mathfrak{S}\left(f, T^{*} g\right)$, maps continuously $H^{1}\left(\mathbb{R}^{n}\right) \times B M O\left(\mathbb{R}^{n}\right)$ into $H^{1}\left(\mathbb{R}^{n}\right)$.

Lemma 7.3. Let b be a non-constant BMO-function and T be a Calderón-Zygmund operator with $T 1=T^{*} 1=0$. Assume that $f \in H_{b}^{1}\left(\mathbb{R}^{n}\right)$ has the wavelet decomposition $f=\sum_{j=1}^{\infty} \sum_{I \subset R_{j}} \sum_{\sigma \in E}\left\langle f, \psi_{I}^{\sigma}\right\rangle \psi_{I}^{\sigma}$, where the $R_{j}$ 's are dyadic cubes and $\sum_{I \subset R_{j}} \sum_{\sigma \in E}\left\langle f, \psi_{I}^{\sigma}\right\rangle \psi_{I}^{\sigma}$ are multiples of $\psi$-atoms related to the cubes $R_{j}$. Set $f_{k}=\sum_{j=1}^{k} \sum_{I \subset R_{j}} \sum_{\sigma \in E}\left\langle f, \psi_{I}^{\sigma}\right\rangle \psi_{I}^{\sigma}, k=1,2, \ldots$. Then, the sequence $\left\{[b, T]\left(f_{k}\right)\right\}_{k \geq 1}$ tends to $[b, T](f)$ in the sense of distributions $\mathcal{S}^{\prime}\left(\mathbb{R}^{n}\right)$.

Proof of Lemma 7.1. We first remark (see [36], Proposition 1) that there exists a constant $C>0$ such that for all dyadic cubes $I, I^{\prime}$ and $\sigma, \sigma^{\prime} \in E$, we have

$$
\max \left\{\left|\left\langle A \psi_{I}^{\sigma}, \psi_{I^{\prime}}^{\sigma^{\prime}}\right\rangle\right|,\left|\left\langle B \psi_{I}^{\sigma}, \psi_{I^{\prime}}^{\sigma^{\prime}}\right\rangle\right|\right\} \leq C 2^{-\left|j-j^{\prime}\right|(\delta+n / 2)}\left(\frac{2^{-j}+2^{-j^{\prime}}}{2^{-j}+2^{-j^{\prime}}+\left|x_{I}-x_{I^{\prime}}\right|}\right)^{n+\delta} .
$$

Consequently,

$$
\max \left\{\left|\left\langle A \psi_{I}^{\sigma}, \psi_{I^{\prime}}^{\sigma^{\prime}}\right\rangle\right|,\left|\left\langle B \psi_{I}^{\sigma}, \psi_{I^{\prime}}^{\sigma^{\prime}}\right\rangle\right|\right\} \leq C p_{\delta}\left(I, I^{\prime}\right)
$$

with

$$
p_{\delta}\left(I, I^{\prime}\right)=\frac{2^{-\left|j-j^{\prime}\right|(\delta / 2+n / 2)}}{1+\left|j-j^{\prime}\right|^{2}}\left(\frac{2^{-j}+2^{-j^{\prime}}}{2^{-j}+2^{-j^{\prime}}+\left|x_{I}-x_{I^{\prime}}\right|}\right)^{n+\delta / 2} .
$$


Here $|I|=2^{-j n}$ and $\left|I^{\prime}\right|=2^{-j^{\prime} n}$, while $x_{I}$ and $x_{I^{\prime}}$ denote the centers of the two cubes. On the other hand, it follows from Lemma 1.3 in 14 that there exists a constant $C=C(n, \delta)>0$ such that

$$
\sum_{I^{\prime \prime}} p_{\delta}\left(I, I^{\prime \prime}\right) p_{\delta}\left(I^{\prime}, I^{\prime \prime}\right) \leq C p_{\delta}\left(I, I^{\prime}\right)
$$

Combining (7.2) and (7.3), we obtain

$$
\begin{gathered}
\sum_{I, I^{\prime}, I^{\prime \prime}} \sum_{\sigma, \sigma^{\prime}, \sigma^{\prime \prime} \in E}\left|\left\langle f, \psi_{I}^{\sigma}\right\rangle\left\langle g, \psi_{I^{\prime}}^{\sigma^{\prime}}\right\rangle\left\langle A \psi_{I}^{\sigma}, \psi_{I^{\prime \prime}}^{\sigma^{\prime \prime}}\right\rangle\left\langle B \psi_{I^{\prime}}^{\sigma^{\prime}}, \psi_{I^{\prime \prime}}^{\sigma^{\prime \prime}}\right\rangle\right| \\
\leq C \sum_{I, I^{\prime}} \sum_{\sigma, \sigma^{\prime} \in E} p_{\delta}\left(I, I^{\prime}\right)\left|\left\langle f, \psi_{I}^{\sigma}\right\rangle\right|\left|\left\langle g, \psi_{I^{\prime}}^{\sigma^{\prime}}\right\rangle\right| .
\end{gathered}
$$

It is easy to establish that the matrix $\left\{p_{\delta}\left(I, I^{\prime}\right)\right\}_{I, I^{\prime}}$ is almost diagonal (by taking $\varepsilon=\delta / 4$ in the definition (3.1) of Frazier and Jawerth [16]) and thus is bounded on $\dot{f}_{1}^{0,2}$, the space of all sequences $\left(a_{I}\right)_{I}$ such that $\left(\sum_{I}\left|a_{I}\right|^{2}|I|^{-1} \chi_{I}\right)^{1 / 2}$ is in $L^{1}\left(\mathbb{R}^{n}\right)$. We then use the wavelet characterization of $H^{1}\left(\mathbb{R}^{n}\right)$ (see Theorem 2.1) and the fact that (cf. [16])

$$
\sum_{I^{\prime}} \sum_{\sigma^{\prime} \in E}\left|\left\langle h, \psi_{I^{\prime}}^{\sigma^{\prime}}\right\rangle\left\|\left\langle g, \psi_{I^{\prime}}^{\sigma^{\prime}}\right\rangle \mid \leq C\right\| h\left\|_{H^{1}}\right\| g \|_{B M O}\right.
$$

for all $h \in H^{1}\left(\mathbb{R}^{n}\right)$, to conclude that

$$
\sum_{I, I^{\prime}, I^{\prime \prime}} \sum_{\sigma, \sigma^{\prime}, \sigma^{\prime \prime} \in E}\left|\left\langle f, \psi_{I}^{\sigma}\right\rangle\left\langle g, \psi_{I^{\prime}}^{\sigma^{\prime}}\right\rangle\left\langle A \psi_{I}^{\sigma}, \psi_{I^{\prime \prime}}^{\sigma^{\prime \prime}}\right\rangle\left\langle B \psi_{I^{\prime}}^{\sigma^{\prime}}, \psi_{I^{\prime \prime}}^{\sigma^{\prime \prime}}\right\rangle\right| \leq C\|f\|_{H^{1}}\|g\|_{B M O}
$$

Proof of Lemma 7.2, By Lemma 7.1, we have

$$
\begin{aligned}
\mathfrak{P}(f, g) & =\sum_{i=1}^{K} \mathfrak{S}\left(A_{i} f, B_{i} g\right) \\
& =\sum_{i=1}^{K} \sum_{I, I^{\prime}, I^{\prime \prime}} \sum_{\sigma, \sigma^{\prime}, \sigma^{\prime \prime} \in E}\left\langle f, \psi_{I}^{\sigma}\right\rangle\left\langle g, \psi_{I^{\prime}}^{\sigma^{\prime}}\right\rangle\left\langle A_{i} \psi_{I}^{\sigma}, \psi_{I^{\prime \prime}}^{\sigma^{\prime \prime}}\right\rangle\left\langle B_{i} \psi_{I^{\prime}}^{\sigma^{\prime}}, \psi_{I^{\prime \prime}}^{\sigma^{\prime \prime}}\right\rangle\left(\psi_{I^{\prime \prime}}^{\sigma^{\prime \prime}}\right)^{2},
\end{aligned}
$$

where all the series converge in $L^{1}\left(\mathbb{R}^{n}\right)$. For any dyadic cubes $I, I^{\prime}, \sigma, \sigma^{\prime} \in E$, we have

$$
\begin{aligned}
& \sum_{i=1}^{K} \sum_{I^{\prime \prime}} \sum_{\sigma^{\prime \prime} \in E}\left\langle f, \psi_{I}^{\sigma}\right\rangle\left\langle g, \psi_{I^{\prime}}^{\sigma^{\prime}}\right\rangle\left\langle A_{i} \psi_{I}^{\sigma}, \psi_{I^{\prime \prime}}^{\sigma^{\prime \prime}}\right\rangle\left\langle B_{i} \psi_{I^{\prime}}^{\sigma^{\prime}}, \psi_{I^{\prime \prime}}^{\sigma^{\prime \prime}}\right\rangle\left(\psi_{I^{\prime \prime}}^{\sigma^{\prime \prime}}\right)^{2} \\
& \quad=\sum_{i=1}^{K} \sum_{I^{\prime \prime}} \sum_{\sigma^{\prime \prime} \in E}\left\langle f, \psi_{I}^{\sigma}\right\rangle\left\langle g, \psi_{I^{\prime}}^{\sigma^{\prime}}\right\rangle\left\langle A_{i} \psi_{I}^{\sigma}, \psi_{I^{\prime \prime}}^{\sigma^{\prime \prime}}\right\rangle\left\langle B_{i} \psi_{I^{\prime}}^{\sigma^{\prime}}, \psi_{I^{\prime \prime}}^{\sigma^{\prime \prime}}\right\rangle\left(\left(\psi_{I^{\prime \prime}}^{\sigma^{\prime \prime}}\right)^{2}-\left(\psi_{I}^{\sigma}\right)^{2}\right)
\end{aligned}
$$

since (see Remark 4.1)

$$
\sum_{i=1}^{K} \sum_{I^{\prime \prime}} \sum_{\sigma^{\prime \prime} \in E}\left\langle A_{i} \psi_{I}^{\sigma}, \psi_{I^{\prime \prime}}^{\sigma^{\prime \prime}}\right\rangle\left\langle B_{i} \psi_{I^{\prime}}^{\sigma^{\prime}}, \psi_{I^{\prime \prime}}^{\sigma^{\prime \prime}}\right\rangle=\int_{\mathbb{R}^{n}}\left(\sum_{i=1}^{K} A_{i} \psi_{I}^{\sigma} \cdot B_{i} \psi_{I^{\prime}}^{\sigma^{\prime}}\right) d x=0 .
$$


An explicit computation gives that $\left|\psi_{I^{\prime \prime}}^{\sigma^{\prime \prime}}\right|^{2}-\left|\psi_{I}^{\sigma}\right|^{2}$ is in $H^{1}\left(\mathbb{R}^{n}\right)$, with

$$
\left\|\left|\psi_{I^{\prime \prime}}^{\sigma^{\prime \prime}}\right|^{2}-\left|\psi_{I}^{\sigma}\right|^{2}\right\|_{H^{1}} \leq C\left(\log \left(2^{-j}+2^{-j^{\prime \prime}}\right)^{-1}+\log \left(\left|x_{I}-x_{I^{\prime \prime}}\right|+2^{-j}+2^{-j^{\prime \prime}}\right)\right) .
$$

Here $|I|=2^{-j n}$ and $\left|I^{\prime \prime}\right|=2^{-j^{\prime \prime} n}$, while $x_{I}$ and $x_{I^{\prime \prime}}$ denote the centers of the two cubes. Consequently, by (7.1) and (7.3), we get

$$
\begin{aligned}
& \left\|\sum_{i=1}^{K} \sum_{I^{\prime \prime}} \sum_{\sigma^{\prime \prime} \in E}\left\langle f, \psi_{I}^{\sigma}\right\rangle\left\langle g, \psi_{I^{\prime}}^{\sigma^{\prime}}\right\rangle\left\langle A_{i} \psi_{I}^{\sigma}, \psi_{I^{\prime \prime}}^{\sigma^{\prime \prime}}\right\rangle\left\langle B_{i} \psi_{I^{\prime}}^{\sigma^{\prime}}, \psi_{I^{\prime \prime}}^{\sigma^{\prime \prime}}\right\rangle\left(\psi_{I^{\prime \prime}}^{\sigma^{\prime \prime}}\right)^{2}\right\|_{H^{1}} \\
& \quad \leq \sum_{i=1}^{K} \sum_{I^{\prime \prime}} \sum_{\sigma^{\prime \prime} \in E}\left|\left\langle f, \psi_{I}^{\sigma}\right\rangle\left\langle g, \psi_{I^{\prime}}^{\sigma^{\prime}}\right\rangle\left\langle A_{i} \psi_{I}^{\sigma}, \psi_{I^{\prime \prime}}^{\sigma^{\prime \prime}}\right\rangle\left\langle B_{i} \psi_{I^{\prime}}^{\sigma^{\prime}}, \psi_{I^{\prime \prime}}^{\sigma^{\prime \prime}}\right\rangle\right|\left\|\left(\psi_{I^{\prime \prime}}^{\sigma^{\prime \prime}}\right)^{2}-\left(\psi_{I}^{\sigma}\right)^{2}\right\|_{H^{1}} \\
& \quad \leq C \sum_{i=1}^{K} \sum_{I^{\prime \prime}} \sum_{\sigma^{\prime \prime} \in E}\left|\left\langle f, \psi_{I}^{\sigma}\right\rangle\left\langle g, \psi_{I^{\prime}}^{\sigma^{\prime}}\right\rangle\right| p_{\delta}\left(I, I^{\prime \prime}\right) p_{\delta}\left(I^{\prime}, I^{\prime \prime}\right) \\
& \quad \leq C p_{\delta}\left(I, I^{\prime}\right)\left|\left\langle f, \psi_{I}^{\sigma}\right\rangle\right|\left\langle g, \psi_{I^{\prime}}^{\sigma^{\prime}}\right\rangle \mid ;
\end{aligned}
$$

here we used the fact that

$$
\begin{aligned}
& \left(1+\left|j-j^{\prime \prime}\right|^{2}\right) \log \left(\frac{\left|x_{I}-x_{I^{\prime \prime}}\right|+2^{-j}+2^{-j^{\prime \prime}}}{2^{-j}+2^{-j^{\prime \prime}}}\right) \\
& \quad \leq C(\delta) 2^{\left|j-j^{\prime \prime}\right| \delta / 2}\left(\frac{\left|x_{I}-x_{I^{\prime \prime}}\right|+2^{-j}+2^{-j^{\prime \prime}}}{2^{-j}+2^{-j^{\prime \prime}}}\right)^{\delta / 2} .
\end{aligned}
$$

Thus, the same argument as in the proof of Lemma 7.1 allows us to conclude that

$$
\begin{aligned}
\|\mathfrak{P}(f, g)\|_{H^{1}} & \leq C \sum_{I, I^{\prime}} \sum_{\sigma, \sigma^{\prime} \in E} p_{\delta}\left(I, I^{\prime}\right)\left|\left\langle f, \psi_{I}^{\sigma}\right\rangle \|\left\langle g, \psi_{I^{\prime}}^{\sigma^{\prime}}\right\rangle\right| \\
& \leq C\|f\|_{H^{1}}\|g\|_{B M O},
\end{aligned}
$$

which ends the proof.

Before giving the proof of Lemma 7.3, let us recall the following lemma. It can be found in [17].

Lemma A (see 17, Lemma 2.3). Let T be a Calderón-Zygmund operator satisfying $T 1=0$. Then $T$ maps $\mathcal{S}\left(\mathbb{R}^{n}\right)$ into $L^{\infty}\left(\mathbb{R}^{n}\right)$. Moreover, there exists a constant $C>0$, depending only on $T$, such that for any $\phi \in \mathcal{S}\left(\mathbb{R}^{n}\right)$ with $\operatorname{supp} \phi \subset B\left(x_{0}, r\right)$, we have

$$
\|T \phi\|_{L^{\infty}} \leq C\left(\|\phi\|_{L^{\infty}}+r\||\nabla \phi|\|_{L^{\infty}}\right) .
$$

Proof of Lemma 7.3 . By Theorem 3.2 it is sufficient to prove that

$$
\lim _{k \rightarrow \infty} \int_{\mathbb{R}^{n}} T\left(\mathfrak{S}\left(f_{k}, b\right)\right) h d x=\int_{\mathbb{R}^{n}} T(\mathfrak{S}(f, b)) h d x,
$$

for all $h \in \mathcal{S}\left(\mathbb{R}^{n}\right)$. Because of the hypothesis, we observe that $\mathfrak{S}(f, b) \in H^{1}\left(\mathbb{R}^{n}\right)$ and $\mathfrak{S}\left(f_{k}, b\right) \in L^{q}\left(\mathbb{R}^{n}\right), k=1,2, \ldots$, for some $q \in(1,2)$ (see Lemma 2.1). 
Let $\mathfrak{S}(f, b)=\sum_{j=1}^{\infty} \lambda_{j} a_{j}$ be a classical $L^{q}$-atomic decomposition of $\mathfrak{S}(f, b)$. Then, $T\left(\sum_{j=1}^{k} \lambda_{j} a_{j}\right)$ tends to $T(\mathfrak{S}(f, b))$ in $L^{1}\left(\mathbb{R}^{n}\right)$ (in fact, it also holds in $H^{1}\left(\mathbb{R}^{n}\right)$ since $\left.T^{*} 1=0\right)$. Hence, as $h \in \mathcal{S}\left(\mathbb{R}^{n}\right) \subset L^{\infty}\left(\mathbb{R}^{n}\right) \cap L^{q^{\prime}}\left(\mathbb{R}^{n}\right)$, where $1 / q+1 / q^{\prime}=1$, $\mathfrak{S}\left(f_{k}, b\right), a_{j} \in L^{q}\left(\mathbb{R}^{n}\right)$ and $T^{*} h \in L^{\infty}\left(\mathbb{R}^{n}\right)$ since $T^{*} 1=0$ (see Lemma A), by Theorem A we get

$$
\begin{aligned}
\int_{\mathbb{R}^{n}} T(\mathfrak{S}(f, b)) h d x & =\lim _{k \rightarrow \infty} \int_{\mathbb{R}^{n}} T\left(\sum_{j=1}^{k} \lambda_{j} a_{j}\right) h d x=\lim _{k \rightarrow \infty} \int_{\mathbb{R}^{n}}\left(\sum_{j=1}^{k} \lambda_{j} a_{j}\right) T^{*} h d x \\
& =\int_{\mathbb{R}^{n}} \mathfrak{S}(f, b) T^{*} h d x=\lim _{k \rightarrow \infty} \int_{\mathbb{R}^{n}} \mathfrak{S}\left(f_{k}, b\right) T^{*} h d x \\
& =\lim _{k \rightarrow \infty} \int_{\mathbb{R}^{n}} T\left(\mathfrak{S}\left(f_{k}, b\right)\right) h d x,
\end{aligned}
$$

since $\mathfrak{S}\left(f_{k}, b\right)$ tends to $\mathfrak{S}(f, b)$ in $L^{1}\left(\mathbb{R}^{n}\right)$ as $f_{k}$ tends to $f$ in $H^{1}\left(\mathbb{R}^{n}\right)$ (see Theorem 3.2). This finishes the proof.

Proof of Theorem 3.4. Let $(f, g) \in H^{1}\left(\mathbb{R}^{n}\right) \times B M O\left(\mathbb{R}^{n}\right)$. By Theorem 3.2 and Lemma 7.2, we obtain $\mathfrak{T}(f, g)=\sum_{i=1}^{K}\left[B_{i} g, T\right]\left(A_{i} f\right) \in L^{1}\left(\mathbb{R}^{n}\right)$; moreover,

$$
\begin{aligned}
\|\mathfrak{T}(f, g)\|_{L^{1}} & \leq \sum_{i=1}^{K}\left\|\mathfrak{R}\left(A_{i} f, B_{i} g\right)\right\|_{L^{1}}+\left\|T\left(\sum_{i=1}^{K} \mathfrak{S}\left(A_{i} f, B_{i} g\right)\right)\right\|_{L^{1}} \\
& \leq C \sum_{i=1}^{K}\left\|A_{i} f\right\|_{H^{1}}\left\|B_{i} g\right\|_{B M O}+\|T\|_{H^{1} \rightarrow L^{1}}\left\|\sum_{i=1}^{K} \mathfrak{S}\left(A_{i} f, B_{i} g\right)\right\|_{H^{1}} \\
& \leq C\|f\|_{H^{1}}\|g\|_{B M O} .
\end{aligned}
$$

This completes the proof.

Proof of Theorem 3.5. Letting $f \in H_{b}^{1}\left(\mathbb{R}^{n}\right)$, we prove $[b, T](f) \in h^{1}\left(\mathbb{R}^{n}\right)$ using the fact that $B M O^{\log }\left(\mathbb{R}^{n}\right)$ is the dual of $H^{\log }\left(\mathbb{R}^{n}\right)$ (see [23]). Indeed, by Theorem 2.2 there exists a decomposition $f=\sum_{j=1}^{\infty} \sum_{I \subset R_{j}} \sum_{\sigma \in E}\left\langle f, \psi_{I}^{\sigma}\right\rangle \psi_{I}^{\sigma}$, where $\sum_{I \subset R_{j}} \sum_{\sigma \in E}\left\langle f, \psi_{I}^{\sigma}\right\rangle \psi_{I}^{\sigma}$ are multiples of $\psi$-atoms related to the dyadic cubes $R_{j}$. Set $f_{k}=\sum_{j=1}^{k} \sum_{I \subset R_{j}} \sum_{\sigma \in E}\left\langle f, \psi_{I}^{\sigma}\right\rangle \psi_{I}^{\sigma}, k=1,2, \ldots$. Then, the sequence $[b, T]\left(f_{k}\right)$ tends to $[b, T](f)$ in the sense of distributions $\mathcal{S}^{\prime}\left(\mathbb{R}^{n}\right)$ (see Lemma 7.3), and thus

$$
\lim _{k \rightarrow \infty} \int_{\mathbb{R}^{n}}[b, T]\left(f_{k}\right) h d x=\int_{\mathbb{R}^{n}}[b, T](f) h d x
$$

for all $h \in C_{0}^{\infty}\left(\mathbb{R}^{n}\right)$. Notice that $[b, T]\left(f_{k}\right) \in L^{2}\left(\mathbb{R}^{n}\right)$ and $[b, T](f) \in L^{1}\left(\mathbb{R}^{n}\right)$. 
Let $h \in C_{0}^{\infty}\left(\mathbb{R}^{n}\right)$. By Lemma 4.2. Lemma 4.3. Lemma 4.4, Remark 4.1 and Corollary [7.1. we have $h T\left(f_{k}\right)-f_{k}\left(T^{*} h-\left(T^{*} h\right)_{\mathbb{Q}}\right) \in H^{\log }\left(\mathbb{R}^{n}\right)$. More precisely,

$$
\begin{gathered}
\left\|h T\left(f_{k}\right)-f_{k}\left(T^{*} h-\left(T^{*} h\right)_{\mathbb{Q}}\right)\right\|_{H^{\log }} \\
\leq C\left\{\left\|\mathfrak{S}\left(T\left(f_{k}\right), h\right)-\mathfrak{S}\left(f_{k}, T^{*} h-\left(T^{*} h\right)_{\mathbb{Q}}\right)\right\|_{H^{1}}\right. \\
\quad+\sum_{j=1,4}\left(\left\|\Pi_{j}\left(T\left(f_{k}\right), h\right)\right\|_{H^{1}}+\left\|\Pi_{j}\left(f_{k}, T^{*} h-\left(T^{*} h\right)_{\mathbb{Q}}\right)\right\|_{H^{1}}\right) \\
\left.+\left\|\Pi_{2}\left(T\left(f_{k}\right), h\right)\right\|_{H^{\log }}+\left\|\Pi_{2}\left(f_{k}, T^{*} h-\left(T^{*} h\right)_{\mathbb{Q}}\right)\right\|_{H^{\log }}\right\} \\
\leq C\left\{\left\|f_{k}\right\|_{H^{1}}\|h\|_{B M O}+\left\|T\left(f_{k}\right)\right\|_{H^{1}}\|h\|_{B M O}+\left\|f_{k}\right\|_{H^{1}}\left\|T^{*} h-\left(T^{*} h\right)_{\mathbb{Q}}\right\|_{B M O}\right. \\
\left.+\left\|T\left(f_{k}\right)\right\|_{H^{1}}\|h\|_{B M O^{+}}+\left\|f_{k}\right\|_{H^{1}}\left\|T^{*} h-\left(T^{*} h\right)_{\mathbb{Q}}\right\|_{B M O^{+}}\right\} \\
\leq C\left(\left\|f_{k}\right\|_{H^{1}}\|h\|_{b m o}+\left\|f_{k}\right\|_{H^{1}}\left\|T^{*} h\right\|_{B M O}\right) \leq C\|f\|_{H^{1}}\|h\|_{b m o} ;
\end{gathered}
$$

here one used $\mathfrak{S}\left(f, T^{*} h-\left(T^{*} h\right)_{\mathbb{Q}}\right)=\mathfrak{S}\left(f, T^{*} h\right),\left\|T^{*} h-\left(T^{*} h\right)_{\mathbb{Q}}\right\|_{B M O^{+}}=$ $\left\|T^{*} h\right\|_{B M O}$ and $\left\|f_{k}\right\|_{H^{1}} \leq C\|f\|_{H^{1}}$. As the $L^{2}$-functions $f_{k}$ have compact support, $b \in B M O^{\log }\left(\mathbb{R}^{n}\right) \subset B M O\left(\mathbb{R}^{n}\right)$, we deduce that $b h T\left(f_{k}\right), h T\left(b f_{k}\right), b f_{k} T^{*} h \in$ $L^{1}\left(\mathbb{R}^{n}\right)$. Moreover, $\int_{\mathbb{R}^{n}} h T\left(b f_{k}\right) d x=\int_{\mathbb{R}^{n}} b f_{k} T^{*} h d x$ since $h T\left(b f_{k}\right)-b f_{k} T^{*} h \in$ $H^{1}\left(\mathbb{R}^{n}\right)$ (see Theorem A). Therefore, as $B M O^{\log }\left(\mathbb{R}^{n}\right)$ is the dual of $H^{\log }\left(\mathbb{R}^{n}\right)$ (see 23]), we get

$$
\begin{aligned}
& \left|\int_{\mathbb{R}^{n}}[b, T]\left(f_{k}\right) h d x\right|=\left|\int_{\mathbb{R}^{n}} b\left(h T\left(f_{k}\right)-f_{k} T^{*} h\right) d x\right| \\
& \leq\left|\int_{\mathbb{R}^{n}} b\left(h T\left(f_{k}\right)-f_{k}\left(T^{*} h-\left(T^{*} h\right)_{\mathbb{Q}}\right)\right) d x\right|+\left|\left(T^{*} h\right)_{\mathbb{Q}}\right|\left|\int_{\mathbb{R}^{n}} b f_{k} d x\right| \\
& \leq C\|b\|_{B M O^{\log }}\left\|h T\left(f_{k}\right)-f_{k}\left(T^{*} h-\left(T^{*} h\right)_{\mathbb{Q}}\right)\right\|_{H^{\log }}+\left|\left(T^{*} h\right)_{\mathbb{Q}}\right|\left|\int_{\mathbb{R}^{n}} b f_{k} d x\right| \\
& \leq C\|b\|_{B M O^{\log }}\|f\|_{H^{1}}\|h\|_{b m o}+\left|\left(T^{*} h\right)_{\mathbb{Q}}\right|\left|\sum_{j=1}^{k} \sum_{I \subset R_{j}} \sum_{\sigma \in E}\left\langle f, \psi_{I}^{\sigma}\right\rangle\left\langle b, \psi_{I}^{\sigma}\right\rangle\right| .
\end{aligned}
$$

The above inequality and (7.4) imply that for all $h \in C_{0}^{\infty}\left(\mathbb{R}^{n}\right)$, we obtain

$$
\left|\int_{\mathbb{R}^{n}}[b, T](f) h d x\right| \leq C\|b\|_{B M O^{\log }}\|f\|_{H^{1}}\|h\|_{b m o}
$$

since $\mathfrak{S}(f, b) \in H^{1}\left(\mathbb{R}^{n}\right)$ (see Theorem 5.1) and thus (see Remark 4.1)

$$
\lim _{k \rightarrow \infty} \sum_{j=1}^{k} \sum_{I \subset R_{j}} \sum_{\sigma \in E}\left\langle f, \psi_{I}^{\sigma}\right\rangle\left\langle b, \psi_{I}^{\sigma}\right\rangle=\int_{\mathbb{R}^{n}} \mathfrak{S}(f, b) d x=0 .
$$


This proves that $[b, T](f) \in h^{1}\left(\mathbb{R}^{n}\right)$ since $h^{1}\left(\mathbb{R}^{n}\right)$ is the dual of $v m o\left(\mathbb{R}^{n}\right)$ (see Section 2). Furthermore,

$$
\|[b, T](f)\|_{h^{1}} \leq C\|b\|_{B M O^{\log }}\|f\|_{H^{1}} \leq C\|b\|_{B M O^{\log }}\|b\|_{B M O}^{-1}\|f\|_{H_{b}^{1}},
$$

which ends the proof of Theorem 3.5 .

Proof of Theorem 3.6. By Theorem 3.2 and Theorem 5.1 together with Lemma 4.2 and Lemma 4.3, it is sufficient to prove that the linear operator

$$
f \mapsto \mathfrak{U}(f, b):=b T f-T\left(\Pi_{2}(f, b)\right)
$$

is bounded from $H^{1}\left(\mathbb{R}^{n}\right)$ into itself. Similarly to the proof of Theorem 3.1, we first consider $f$ a $\psi$-atom related to the cube $Q=Q\left[x_{0}, r\right]$ and note that

$$
\mathfrak{U}(f, b)=\mathfrak{U}\left(f, b-b_{Q}\right)=\left(b-b_{Q}\right) T f-T\left(\Pi_{2}\left(f, b-b_{Q}\right)\right) .
$$

Letting $\varepsilon \in(0,1)$, recall that (see [41]) $g$ is an $\varepsilon$-molecule for $H^{1}\left(\mathbb{R}^{n}\right)$ centered at $y_{0}$ if

$$
\int_{\mathbb{R}^{n}} g(x) d x=0 \quad \text { and } \quad\|g\|_{L^{q}}^{1 / 2}\left\|g\left|\cdot-y_{0}\right|^{2 n \varepsilon}\right\|_{L^{q}}^{1 / 2}=: \mathfrak{N}(g)<\infty,
$$

where $q=1 /(1-\varepsilon)$. It is well known that if $g$ is an $\varepsilon$-molecule for $H^{1}\left(\mathbb{R}^{n}\right)$ centered at $y_{0}$, then $g \in H^{1}\left(\mathbb{R}^{n}\right)$ and $\|g\|_{H^{1}} \leq C \mathfrak{N}(g)$, where $C>0$ depends only on $n, \varepsilon$.

We now prove that $\left(b-b_{Q}\right) T f$ is an $\varepsilon$-molecule for $H^{1}\left(\mathbb{R}^{n}\right)$ centered at $x_{0}$ when $T$ is a $\delta$-Calderón-Zygmund operator for some $\delta \in(0,1]$ and $\varepsilon=\delta /(4 n)<1 / 2$. Note first that $f$ is $C$ times a classical $L^{2}$-atom related to the cube $m Q$. It is clear that $\int_{\mathbb{R}^{n}}\left(b-b_{Q}\right) T f d x=0$ since $T^{*} 1=T^{*} b=0$. As $q=1 /(1-\varepsilon)<2$, the fact that $\left|b_{Q}-b_{2 m Q}\right| \leq C\|b\|_{B M O}$, together with the Hölder inequality and the John-Nirenberg inequality, gives

$$
\left\|\left(b-b_{Q}\right) T f \cdot \chi_{2 m Q}\right\|_{L^{q}} \leq C|Q|^{1 / q-1}\|b\|_{B M O} .
$$

It is well known that $|T f(x)| \leq C \frac{r^{\delta}}{\left|x-x_{0}\right|^{n+\delta}}$, for all $x \in(2 m Q)^{c}$, since $T$ is a $\delta$-Calderón-Zygmund operator. Hence

$$
\begin{aligned}
\left\|\left(b-b_{Q}\right) T f \cdot \chi_{(2 m Q)^{c}}\right\|_{L^{q}} & \leq C\left(\int_{(2 m Q)^{c}}\left|b-b_{Q}\right|^{q}\left(\frac{r^{\delta}}{\left|x-x_{0}\right|^{n+\delta}}\right)^{q} d x\right)^{1 / q} \\
& \leq C|Q|^{1 / q-1}\|b\|_{B M O} .
\end{aligned}
$$

The last inequality, which can be found in [40], is classical. Combining this and (7.6), we obtain

$$
\left\|\left(b-b_{Q}\right) T f\right\|_{L^{q}} \leq C|Q|^{1 / q-1}\|b\|_{B M O} .
$$

Similarly, we also have

$$
\left\|\left(b-b_{Q}\right) T f .\left|\cdot-x_{0}\right|^{2 n \varepsilon} \cdot \chi_{2 m Q}\right\|_{L^{q}} \leq C|Q|^{2 \varepsilon+1 / q-1}\|b\|_{B M O}
$$

and as $2 n \varepsilon=\delta / 2$,

$$
\begin{aligned}
\left\|\left(b-b_{Q}\right) T f .\left|\cdot-x_{0}\right|^{2 n \varepsilon} \cdot \chi_{(2 m Q)^{c}}\right\|_{L^{q}} & \leq C\left(\int_{(2 m Q)^{c}}\left|b-b_{Q}\right|^{q}\left(\frac{r^{\delta}}{\left|x-x_{0}\right|^{n+\delta / 2}}\right)^{q} d x\right)^{1 / q} \\
& \leq C|Q|^{2 \varepsilon+1 / q-1}\|b\|_{B M O} .
\end{aligned}
$$

Consequently,

$$
\left\|\left(b-b_{Q}\right) T f .\left|\cdot-x_{0}\right|^{2 n \varepsilon}\right\|_{L^{q}} \leq C|Q|^{2 \varepsilon+1 / q-1}\|b\|_{B M O} .
$$


Combining this and (7.7), we get that $\left(b-b_{Q}\right) T f$ is an $\varepsilon$-molecule for $H^{1}\left(\mathbb{R}^{n}\right)$ centered at $x_{0}$; moreover,

$$
\mathfrak{N}\left(\left(b-b_{Q}\right) T f\right) \leq C|Q|^{\varepsilon+1 / q-1}\|b\|_{B M O} \leq C\|b\|_{B M O},
$$

since $q=1 /(1-\varepsilon)$. Thus, by (7.5) and Remark 4.1,

$$
\|\mathfrak{U}(f, b)\|_{H^{1}} \leq C \mathfrak{N}\left(\left(b-b_{Q}\right) T f\right)+\left\|T\left(\Pi_{2}\left(f, b-b_{Q}\right)\right)\right\|_{H^{1}} \leq C\|b\|_{B M O} .
$$

Now, let us consider $f \in H_{\text {fin }}^{1}\left(\mathbb{R}^{n}\right)$. By Lemma 6.2, there exists a finite decomposition $f=\sum_{j=1}^{k} \lambda_{j} a_{j}$ such that $\sum_{j=1}^{k}\left|\lambda_{j}\right| \leq C\|f\|_{H^{1}}$. Consequently, by (17.8), we obtain that

$$
\|\mathfrak{U}(f, b)\|_{H^{1}} \leq \sum_{j=1}^{k}\left|\lambda_{j}\right|\left\|\mathfrak{U}\left(a_{j}, b\right)\right\|_{H^{1}} \leq C\|f\|_{H^{1}}\|b\|_{B M O},
$$

which ends the proof as $H_{\text {fin }}^{1}\left(\mathbb{R}^{n}\right)$ is dense in $H^{1}\left(\mathbb{R}^{n}\right)$ for the norm $\|\cdot\|_{H^{1}}$.

\section{Commutators of Fractional integrals}

Given $0<\alpha<n$, the fractional integral operator $I_{\alpha}$ is defined by

$$
I_{\alpha} f(x)=\int_{\mathbb{R}^{n}} \frac{f(y)}{|x-y|^{n-\alpha}} d y .
$$

Let $b$ be a locally integrable function. We consider the linear commutator $\left[b, I_{\alpha}\right]$ defined by

$$
\left[b, I_{\alpha}\right](f)=b I_{\alpha} f-I_{\alpha}(b f) .
$$

We end this article by presenting some results related to commutators of fractional integrals as follows.

Theorem 8.1. Let $0<\alpha<n$. There exist a bounded bilinear operator $\mathfrak{R}$ : $H^{1}\left(\mathbb{R}^{n}\right) \times B M O\left(\mathbb{R}^{n}\right) \rightarrow L^{n /(n-\alpha)}\left(\mathbb{R}^{n}\right)$ and a bounded bilinear operator $\mathfrak{S}: H^{1}\left(\mathbb{R}^{n}\right)$ $\times B M O\left(\mathbb{R}^{n}\right) \rightarrow L^{1}\left(\mathbb{R}^{n}\right)$ such that

$$
\left[b, I_{\alpha}\right](f)=\mathfrak{R}(f, b)+I_{\alpha}(\mathfrak{S}(f, b)) .
$$

Corollary 8.1. Let $0<\alpha<n$ and $b \in B M O\left(\mathbb{R}^{n}\right)$. Then, the linear commutator $\left[b, I_{\alpha}\right]$ maps continuously $H^{1}\left(\mathbb{R}^{n}\right)$ into weak- $L^{n /(n-\alpha)}\left(\mathbb{R}^{n}\right)$.

Theorem 8.2. Let $0<\alpha<n, b \in B M O\left(\mathbb{R}^{n}\right)$, and $1<q \leq \infty$. Then, the linear commutator $\left[b, I_{\alpha}\right]$ maps continuously $H_{b}^{1}\left(\mathbb{R}^{n}\right)$ into $L^{n /(n-\alpha)}\left(\mathbb{R}^{n}\right)$.

The results above can be proved similarly to Theorem 3.2 and Theorem 3.3 . We leave the proofs to the interested readers. When $H_{b}^{1}\left(\mathbb{R}^{n}\right)$ is replaced by $\mathcal{H}_{b}^{1}\left(\mathbb{R}^{n}\right)$, Theorem 8.2 was considered by the authors in [13. There, they proved that

$$
\sup \left\{\left\|\left[b, I_{\alpha}\right](a)\right\|_{L^{n /(n-\alpha)}}: a \text { is a }(\infty, b) \text {-atom }\right\}<\infty .
$$

However, as pointed out before, this argument does not suffice to conclude that $\left[b, I_{\alpha}\right]$ is bounded from $\mathcal{H}_{b}^{1}\left(\mathbb{R}^{n}\right)$ into $L^{n /(n-\alpha)}\left(\mathbb{R}^{n}\right)$. 


\section{ACKNOWLEDGEMENTS}

The author would like to thank Professor Aline Bonami for many very valuable suggestions, discussions and advice to improve this paper. Specifically, Theorem 3.6 is an improvement from the previous version through her ideas. The author would also like to thank Professor Sandrine Grellier for many helpful suggestions and her careful reading and revision of the manuscript. The author is deeply indebted to them.

\section{REFERENCES}

[1] J. Alvarez, R.J. Bagby, D.S. Kurtz and C. Pérez, Weighted estimates for commutators of linear operators. Studia Math. 104 (1993), no. 2, 195-209. MR.1211818 (94k:47044)

[2] J. Alvarez and J. Hounie, Estimates for the kernel and continuity properties of pseudodifferential operators. Ark. Mat. 28 (1990), no. 1, 1-22. MR1049640 (91d:35255)

[3] A. Bonami, J. Feuto and S. Grellier, Endpoint for the div-curl lemma in Hardy spaces. Publ. Mat. 54, No. 2 (2010), 341-358. MR 2675927 (2011f:42024)

[4] A. Bonami, S. Grellier and L. D. Ky, Paraproducts and products of functions in $B M O\left(\mathbb{R}^{n}\right)$ and $H^{1}\left(\mathbb{R}^{n}\right)$ through wavelets, to appear in J. Math. Pure Appl., arXiv: 1103.1822.

[5] G. Bourdaud, Remarques sur certains sous-espaces de $B M O\left(\mathbb{R}^{n}\right)$ et de $b m o\left(\mathbb{R}^{n}\right)$. (French) [Remarks on some subspaces of $B M O\left(\mathbb{R}^{n}\right)$ and $\left.b m o\left(\mathbb{R}^{n}\right)\right]$ Ann. Inst. Fourier (Grenoble) 52 (2002), no. 4, 1187-1218. MR1927078 (2003f:42033)

[6] M. Bownik, Boundedness of operators on Hardy spaces via atomic decompositions. Proc. Amer. Math. Soc. 133 (2005), 3535-3542. MR2163588|(2006d:42028)

[7] Y. Chen and Y. Ding, Commutators of Littlewood-Paley operators. Sci. China Ser. A 52 (2009), no. 11, 2493-2505. MR 2566661 (2010k:42023)

[8] R. Coifman and L. Grafakos, Hardy space estimates for multilinear operators. I. Rev. Mat. Iberoamericana 8 (1992), no. 1, 45-67. MR.1178448 (93j:42011)

[9] R. R. Coifman and G. Weiss, Extensions of Hardy spaces and their use in analysis. Bull. Amer. Math. Soc. 83 (1977), 569-645. MR0447954(56:6264)

[10] R. R. Coifman, R. Rochberg and G. Weiss, Factorization theorems for Hardy spaces in several variables. Ann. of Math. (2), 103, 611-635 (1976) MR0412721(54:843)

[11] G. Dafni, Local VMO and weak convergence in $H^{1}$, Canad. Math. Bull. 45 (2002), 46-59. MR.1884133 (2003d:42034)

[12] I. Daubechies, Orthonormal bases of compactly supported wavelets, Comm. Pure Appl. Math. 41 (1988), 909-996. MR951745|(90m:42039)

[13] Y. Ding, S. Z. Lu and P. Zhang, Continuity of higher order commutators on certain Hardy spaces. Acta Math. Sinica. 18 (2002), 391- 404. MR1910975 (2003d:42035)

[14] S. Dobyinsky, La "version ondelettes" du théorème du Jacobien. (French) [The "wavelet version" of the theorem of the Jacobian] Rev. Mat. Iberoamericana 11 (1995), no. 2, 309-333. MR.1344895 (98a:42020)

[15] C. Fefferman and E. M. Stein, $H^{p}$ spaces of several variables. Acta Math. 129 (1972), 137-193. MR0447953 (56:6263)

[16] M. Frazier and B. Jawerth, A discrete transform and decompositions of distribution spaces. J. Funct. Anal. 93 (1990), no. 1, 34-170. MR1070037|(92a:46042)

[17] M. Frazier, R. Torres and G. Weiss, The boundedness of Calderón-Zygmund operators on the spaces $\dot{F}_{p}^{\alpha, q}$. Rev. Mat. Iberoamericana, 4 (1988), no. 1, 41-72. MR 1009119|(90k:42029)

[18] D. Goldberg, A local version of real Hardy spaces, Duke J. Math. 46 (1979), 27-42. MR523600 $(80 \mathrm{~h}: 46052)$

[19] Y. Han, D. Müller and D. Yang, Littlewood-Paley characterizations for Hardy spaces on spaces of homogeneous type. Math. Nachr. 279 (2006), No. 13-14, 1505-537. MR.2269253 (2007g:42035)

[20] E. Harboure, C. Segovia and J. L. Torrea, Boundedness of commutators of fractional and singular integrals for the extreme values of $p$. Illinois J. Math. 41 (1997), 676-700. MR 1468874 (99j:42025)

[21] E. Hernández and G. Weiss, A first course on wavelets, with a foreword by Yves Meyer, Studies in Advanced Mathematics. CRC Press, Boca Raton, FL, 1996. MR1408902 (97i:42015) 
[22] P. W. Jones and J-L. Journé, On weak convergence in $H^{1}\left(\mathbb{R}^{n}\right)$. Proc. Amer. Math. Soc. 120 (1994), no. 1, 137-138. MR.1159172 (94b:42011)

[23] L. D. Ky, New Hardy spaces of Musielak-Orlicz type and boundedness of sublinear operators, submitted, arXiv:1103.3757.

[24] M-Y. Lee, Weighted norm inequalities of Bochner-Riesz means. J. Math. Anal. Appl. 324 (2006), no. 2, 1274-1281. MR2266557 (2007h:42022)

[25] W. Li, S. Lu and H. Mo, The boundedness for commutators of multipliers. Acta Math. Appl. Sin. Engl. Ser. 23 (2007), no. 1, 113-122. MR2300152 (2008h:42033)

[26] Y. Lin and S. Lu, Boundedness of commutators on Hardy-type spaces. Integr. Equ. Oper. Theory 57 (2007), 381-396. MR2307817(2008h:42023)

[27] Z. Liu and S. Z. Lu, Endpoint estimates for commutators of Calderón-Zygmund type operators. Kodai Math. J. 25 (2002), 79-88. MR1891801 (2003m:42040)

[28] L. Z. Liu, Continuity for commutators of Littlewood-Paley operators on certain Hardy spaces. J. Korean Math. Soc. 40 (2003), No. 1, 41-60. MR1945712 (2003k:42036)

[29] L. Z. Liu and S. Z. Lu, Continuity for maximal multilinear Bochner-Riesz operators on Hardy and Herz-Hardy spaces. Acta Math. Sin. (Engl. Ser.) 22 (2006), no. 1, 69-76. MR2200601 (2006k:42028)

[30] Z. Liu, G. Lu and S. Lu, Continuity properties for the maximal operator associated with the commutator of the Bochner-Riesz operator. Publ. Mat. 47 (2003), no. 1, 45-69. MR.1970894 (2004a:42014)

[31] L. Liu and Q. Tong, Continuity for maximal commutator of Bochner-Riesz operators on some weighted Hardy spaces. Int. J. Math. Math. Sci. 2005, no. 2, 195-201. MR2143751 (2006f:42017)

[32] S. Z. Lu, Q. Wu and D. C. Yang, Boundedness of commutators on Hardy type spaces. Sci. China Ser. A. 45 (2002), 984-997. MR.1942912 (2004b:42031)

[33] S. Z. Lu and L. F. Xu, Boundedness of some Marcinkiewicz integral operators related to higher order commutators on Hardy spaces. Acta Math. Sinica 22 (2006), 105-114. MR.2200606 (2006j:42024)

[34] S. Meda, P. Sjögren and M. Vallarino, On the $H^{1}-L^{1}$ boundedness of operators. Proc. Amer. Math. Soc. 136 (2008), 2921-2931. MR2399059 (2009b:42025)

[35] Y. Meyer, Wavelets and operators. Advanced Mathematics. Cambridge University Press, 1992. MR1228209(94f:42001)

[36] Y. Meyer and R. Coifman, Wavelets, Calderón-Zygmund and multilinear operators. Advanced Mathematics. Cambridge University Press, 1997. MR 1456993 (98e:42001)

[37] E. Nakai and K. Yabuta, Pointwise multipliers for functions of bounded mean oscillation. J. Math. Soc. Japan 37 (1985), 207-218. MR780660 (87d:42020)

[38] C. Pérez, Endpoint estimates for commutators of singular integral operators. J. Funct. Anal. 128 (1995), 163-185. MR.1317714 (95j:42011)

[39] J. L. Rubio de Francia, F. J. Ruiz and J. L. Torrea, Calderón-Zygmund theory for operatorvalued kernels. Adv. in Math. 62 (1986), no. 1, 7-48. MR859252 (88f:42035)

[40] E. M. Stein, Harmonic Analysis: Real-Variable Methods, Orthogonality, and Oscillatory Integrals. Princeton Univ. Press, Princeton, NJ, 1993. MR1232192 (95c:42002)

[41] M. H. Taibleson and G. Weiss, The molecular characterization of certain Hardy spaces; Astérisque. 77 (1980), 67-149. MR604370 (83g:42012)

[42] K. Wang and L. Liu, Boundedness for multilinear commutator of multiplier operator on Hardy spaces. Sci. Ser. A Math. Sci. (N.S.) 17 (2009), 19-26. MR2555808 (2011b:42053)

[43] D. Y. Yan, G. E. Hu and J. C. Lan, Weak-type endpoint estimates for multilinear singular integral operators. Acta Math. Sinica 21 (2005), 209- 214. MR2128837 (2005k:42046)

[44] D. Yang and Y. Zhou, A boundedness criterion via atoms for linear operators in Hardy spaces. Constr. Approx. 29, 207-218 (2009). MR2481589 (2010e:42021)

[45] P. Zhang and J. Hua, Commutators of multipliers on Hardy spaces. Anal. Theory Appl. 21 (2005), no. 3, 226-234. MR2319191 (2008b:42021)

Department of Mathematics, University of Quy Nhon, 170 An Duong Vuong Street, Quy Nhon City, Vietnam

E-mail address: dangky@math.cnrs.fr 